\title{
A NON-CLASSICAL INTERNAL POLAR CONTINUUM THEORY FOR FINITE DEFORMATION OF SOLIDS USING FIRST PIOLA-KIRCHHOFF STRESS TENSOR
}

\author{
K. S. SURANA ${ }^{1}$, A. D. JOY ${ }^{1}$, and J. N. REDDY ${ }^{2}$ \\ ${ }^{1}$ Mechanical Engineering \\ University of Kansas \\ Lawrence, Kansas \\ USA \\ email: kssurana@ku.edu \\ ${ }^{2}$ Mechanical Engineering \\ Texas A\&M University \\ College Station, Texas \\ USA
}

\begin{abstract}
This paper presents a non-classical internal polar continuum theory for finite deformation of isotropic, homogeneous compressible and incompressible solid continua. The classical continuum theories only incorporate partial physics of deformation in the thermodynamic framework. Since the Jacobian of deformation $\boldsymbol{J}$ is fundamental measure of deformation in solid continua, $\boldsymbol{J}$ in its entirety must be incorporated in the thermodynamic framework. Polar decomposition of $\boldsymbol{J}$ into right stretch tensor $\boldsymbol{S}_{\boldsymbol{r}}$ and pure rotation tensor $\boldsymbol{R}$ shows that entirety of $\boldsymbol{J}$ implies entirety of $\boldsymbol{S}_{\boldsymbol{r}}$ and $\boldsymbol{R}$. The classical continuum theories for isotropic and homogeneous solid continua are derived purely using $\boldsymbol{S}_{\boldsymbol{r}}$, thus ignoring the influence of $\boldsymbol{R}$ altogether. The purpose of this research is to present a new and more complete thermodynamic framework for
\end{abstract}

2010 Mathematics Subject Classification: 74A35, 74A20.

Keywords and phrases: non-classical continuum theory, internal polar continuum theory, solid continua, Jacobian of deformation, polar decomposition, stretch tensor, rotation tensor, finite deformation.

Received June 21, 2016 
finite deformation of solids that incorporates complete deformation physics described by $\boldsymbol{J}$. This can be accomplished by incorporating the additional physics due to $\boldsymbol{R}$ in the current theories as these theories already contain the physics due to $\boldsymbol{S}_{\boldsymbol{r}}$. We note that the rotation tensor $\boldsymbol{R}$ results due to deformation of solid continua, hence arises in all deforming solid continua. Thus, this theory can be referred to as internal polar non-classical theory for solid continua. The use of internal polar non-classical is appropriate as the theory considers internal rotations .If the varying internal rotations and the rotation rates are resisted by the solid continua, then there must exist internal moments that are conjugate to the rotations which together with rotations and rotation rates can result in additional energy storage, dissipation, and memory. Derivations of conservation and balance laws are presented for internal polar non-classical continuum theory for solid continua for finite deformation. Necessity of additional conservation and balance laws is discussed and their derivations are presented. The resulting mathematical model is compared with the mathematical models resulting from the current continuum theories for finite deformation to illustrate the differences in them due to incorporating the additional physics associated with $\boldsymbol{R}$ and thereby incorporating $\boldsymbol{J}$ in its entirety. The non-classical continuum theory for solid continua presented here is not to be confused with the micropolar theories, stress-couple theories, or strain gradient theories as demonstrated in this paper. The objective of the theory presented here is to present new thermodynamic framework for solid continua with finite deformation that is consistent with the deformation physics, which necessitates that $\boldsymbol{J}$ in its entirety must form the basis for derivation of conservation and balance laws. Since this internal polar non-classical continuum theory considers additional physics due to $\boldsymbol{R}$, the resulting thermodynamic framework is more complete and consistent with the physics of deformation compared to the currently used thermodynamic framework.

\section{Introduction, literature review, and scope of present work}

In Lagrangian description of homogeneous, isotropic deforming solid matter the Jacobian of deformation $(\boldsymbol{J})$ is a fundamental measure of deformation in the solid matter. Thus in a consistent thermodynamic framework for deforming solid continua, the Jacobian of deformation in its entirety must form the basis for the derivation of conservation and balance laws. In general the Jacobian of deformation varies between material points, i.e., it varies between a material point and its neighbors. Additionally measures of finite deformation must be considered. Totality of the Jacobian of deformation can be incorporated in the derivations of conservation and balance laws in perhaps more than one way. However, the simplest approach is to perhaps consider polar decomposition of $\boldsymbol{J}$ into stretch tensor (right $\boldsymbol{S}_{\boldsymbol{r}}$ or left $S_{l}$ ) and pure rotation tensor $\boldsymbol{R}$. If the Jacobian of deformation $\boldsymbol{J}$ varies between the neighboring material points so do $\boldsymbol{S}_{\boldsymbol{r}}$ (or $\boldsymbol{S}_{\boldsymbol{l}}$ ) and $\boldsymbol{R}$. Influence of varying $\boldsymbol{S}_{\boldsymbol{r}}$ between material points for finite strain is incorporated in the current classical continuum theories by considering appropriate strain measures such as Green's strain tensor, a quadratic function of $\boldsymbol{S}_{\boldsymbol{r}}$, while the 
influence of the varying rotation tensor $\boldsymbol{R}$ between the material points is completely ignored.

If the varying rotation (due to varying $\boldsymbol{J}$ ) between neighboring material points is resisted by the solid matter, then it can result in conjugate moment tensor. Varying rotations, rotation rates, and the conjugate moment tensor can result in additional energy storage, dissipation, and memory. This physics exists in all homogeneous, isotropic deforming solid matter and must be considered in the derivation of the conservation and balance laws in addition to $\boldsymbol{S}_{\boldsymbol{r}}$ (or Green's strain) if $\boldsymbol{J}$ in its entirety is to form the basis for the thermodynamic framework. The rotations represented by $\boldsymbol{R}$ are due to Jacobian of deformation $\boldsymbol{J}$, hence internal to the deforming matter, and completely defined by $\boldsymbol{J}$. Thus the name internal polar non-classical continuum theory for finite deformation. The two most significant aspects of the work presented in this paper are: (i) consideration of finite deformation and (ii) incorporating $\boldsymbol{J}$, i.e., $\boldsymbol{S}_{\boldsymbol{r}}$ and $\boldsymbol{R}$ in their entirety in the derivation of conservation and balance laws.

In recent papers Surana et al. [1,2] presented conservation and balance laws for internal polar non-classical continuum theory for solid matter based on small strain, small deformation assumption. With these assumptions the distinction between coand contravariant bases disappears. Authors also presented comprehensive literature review on related published works. In the following we present a brief literature review on micropolar theories, nonlocal theories, and stress couple theories. A comprehensive treatment of micropolar theories can be found in the works by Eringen [3-11]. The concept of couple stresses is presented by Koiter [12]. Balance laws for micromorphic materials are presented in [13]. The micropolar theories consider micro deformation due to micro constituents in the continuum. In [14-16] by Reddy et al. and [17] by Zang et al. nonlocal theories are presented for bending, buckling and vibration of beams, beams with nanocarbon tubes and bending of plates. The nonlocal effects are believed to be incorporated due to the work presented by Eringen [8] in which definition of a nonlocal stress tensor is introduced through integral relationship using the product of macroscopic stress tensor and a distance kernel representing the nonlocal effects. The polar continuum theory for solid continua presented in this paper is strictly local and non-micropolar. The concept of couple stresses was introduced by Voigt in 1881 by assuming a couple or moment per unit area on the oblique plane of the deformed tetrahedron in addition to the stress or force per unit area. Since the introduction of this concept many published works have appeared. We cite some recent works, most of which are related to micropolar stress couple theories. Authors in [18] 
report experimental study of micropolar and couple stress elasticity of compact bones in bending. Conservation integrals in couple stress elasticity are reported in [19]. A microstructure-dependent Timoshenko beam model based on modified couple stress theories is reported by Ma et al. [20]. Further account of couple stress theories in conjunction with beams can be found in [21-23]. Treatment of rotation gradient dependent strain energy and its specialization to Von Kármán plates and beams can be found in [24]. Other accounts of micropolar elasticity and Cosserat modeling of cellular solids can be found in [25-27]. We remark that in [18-27], Lagrangian description is used for solid matter, however the mathematical descriptions are purely derived using strain energy density functional and principle of virtual work. This approach works well for elastic solids in which mechanical deformation is reversible. Extension of these works to thermoviscoelastic solids with and without memory is not possible. In such materials the thermal field and mechanical deformation are coupled due to the fact that the rate of work results in rate of entropy production. In [28] Altenbach and Eremeyev present a linear theory for micropolar plates. Each material point is regarded as a small rigid body with six degrees of freedom. Kinematics of plates is described using the vector of translations and the vector of rotations as dependent variables. Equations of equilibrium are established in $\mathbb{R}^{3}$ and $\mathbb{R}^{2}$. Strain energy density function is used to present linear constitutive theory. The mathematical models of [29] are extended by the same authors to present strain rate tensors and the constitutive equations for inelastic micropolar materials. In [30], authors consider the conditions for the existence of the acceleration waves in thermoelastic micropolar media. The work concludes that the presence of the energy equation with Fourier heat conduction law does not influence the wave physics in thermoelastic micropolar media. Thus, from the point of view of acceleration waves in thermoelastic polar media, thermal effects, i.e., temperature can be treated as a parameter. In [31], authors present a collection of papers related to the mechanics of continua dealing with micro-macro aspects of the physics (largely related to solid matter). In [32] a micro-polar theory is presented for binary media with applications to phase-transitional flow of fiber suspensions. Such flows take place during the filling state of injection molding of short fiber reinforced thermoplastics. A similarity solution for boundary layer flow of a polar fluid is given in [33]. In specific the paper borrows constitutive equations that are claimed to be valid for flow behavior of a suspension of very fine particles in a viscous fluid. Kinematics of micropolar continuum is presented in [34]. Material symmetry groups for linear Cosserat continuum and non-linear polar elastic continuum are considered in $[35,36]$. 
Grekova et al. [37-39] consider various aspects of wave processes in ferromagnetic medium and elastic medium with micro-rotations as well as some aspects of linear reduced Cosserat medium. In [40-58] various aspects of the kinematics of micropolar theories, stress couple theories, etc. are discussed and presented including some applications to plates and shells.

\section{Notations, definitions, bases, measures, and preliminary considerations}

\subsection{Notations, some basic definitions}

We use an overbar to express quantities in the current configuration in Eulerian description, i.e., all quantities with overbars are functions of deformed coordinates $\bar{x}_{i}$ and time $t$. Quantities without an overbar imply Lagrangian description of the quantities in the current configuration, i.e., these are functions of undeformed coordinates $x_{i}$ and time $t$. We use the configuration at time $t=t_{0}=0$, commencement of evolution, to be the reference configuration. Thus, $x_{i} ; i=1,2,3$ and $\overline{\boldsymbol{x}}$ are the coordinates of a material point in the reference and current configurations, respectively, both measured in a fixed Cartesian $x$-frame. This paper only considers Lagrangian description, hence all measures are expressed in terms of coordinates of the material points in the undeformed configuration (same as reference configuration in the present work) $\boldsymbol{x}$ and time $t$. We use $\boldsymbol{J}=\boldsymbol{e}_{i} \otimes \boldsymbol{e}_{j} \frac{\partial \bar{x}_{j}}{\partial x_{i}}$ or $[J]=\left[\frac{\partial\{\bar{x}\}}{\partial\{x\}}\right]$ to be the Jacobian of deformation, a covariant measure in Lagrangian description. Likewise, $\overline{\boldsymbol{J}}=\boldsymbol{e}_{i} \otimes \boldsymbol{e}_{j} \frac{\partial x_{j}}{\partial \bar{x}_{i}}$ or $[\bar{J}]=\left[\frac{\partial\{x\}}{\partial\{\bar{x}\}}\right]$ is also Jacobian of deformation but it is contravariant measure in Eulerian description.

The existence of varying rotations at the neighboring material points (evident from polar decomposition of the Jacobian of deformation) when resisted by the matter can result in additional energy storage or dissipation in the deforming matter. Just like points of application of forces when displaced result in work, the moments through rotations result in work as well. Thus, in the development of the non-classical internal polar continuum theory presented here we consider existence of internal rotations and moments independent of forces and displacements. Due to finite deformation, undeformed and the corresponding deformed volumes are not the same as $\overline{\boldsymbol{x}} \neq \boldsymbol{x}$. Thus, care is needed in choosing various measures that describe deformation of the solid continua. Consider a volume of matter $\underset{\sim}{V}$ in the reference configuration (Figure 1(a)) with closed boundary $\partial \underset{\sim}{V}$. The volume $V$ is isolated from $\underset{\sim}{V}$ by a hypothetical surface $\partial V$ as in cut principle of Cauchy. Consider a tetrahedron $T_{1}$ shown in Figure 1(a) such that its oblique plane is part of $\partial V$ and its other three planes are orthogonal to each other 
and parallel to the planes of the $x$-frame. Upon deformation $\underset{\sim}{V}$ and $\partial \underset{\sim}{V}$ occupy $\underset{\sim}{\bar{V}}$ and $\partial \bar{\sim}$ and likewise $V$ and $\partial V$ deform into $\bar{V}$ and $\partial \bar{V}$. The tetrahedron $T_{1}$ deforms into $\bar{T}_{1}$ whose edges (under finite deformation) are nonorthogonal covariant base vector $\tilde{\boldsymbol{g}}_{i}$. The plane of the tetrahedron formed by the covariant base vectors are flat but obviously nonorthogonal to each other. We assume the tetrahedron to be the small neighborhood of material point $\bar{o}$ so that assumption of the oblique plane $\bar{A} \bar{B} \bar{C}$ being flat but still part of $\partial \bar{V}$ is valid. When the deformed tetrahedron is isolated from volume $\bar{V}$ it must be in equilibrium under the action of disturbance on the surface of $\bar{A} \bar{B} \bar{C}$ from the volume surrounding $\bar{V}$ and the internal fields that act on the flat faces which equilibrate with the mating faces in volume $\bar{V}$ when the tetrahedron $\bar{T}_{1}$ is placed back in the volume $\bar{V}$. Consider deformed tetrahedron $\bar{T}_{1}$. Let $\overline{\boldsymbol{P}}$ be the average stress on plane $\bar{A} \bar{B} \bar{C}, \overline{\boldsymbol{M}}$ be the average moment per unit area also on plane $\bar{A} \bar{B} \bar{C}$ henceforth referred to as moment for short and $\overline{\boldsymbol{n}}$ be the normal to the face $\bar{A} \bar{B} \overline{\boldsymbol{C}} . \overline{\boldsymbol{P}}, \overline{\boldsymbol{M}}, \overline{\boldsymbol{n}}$ all have different directions when the deformation is finite.
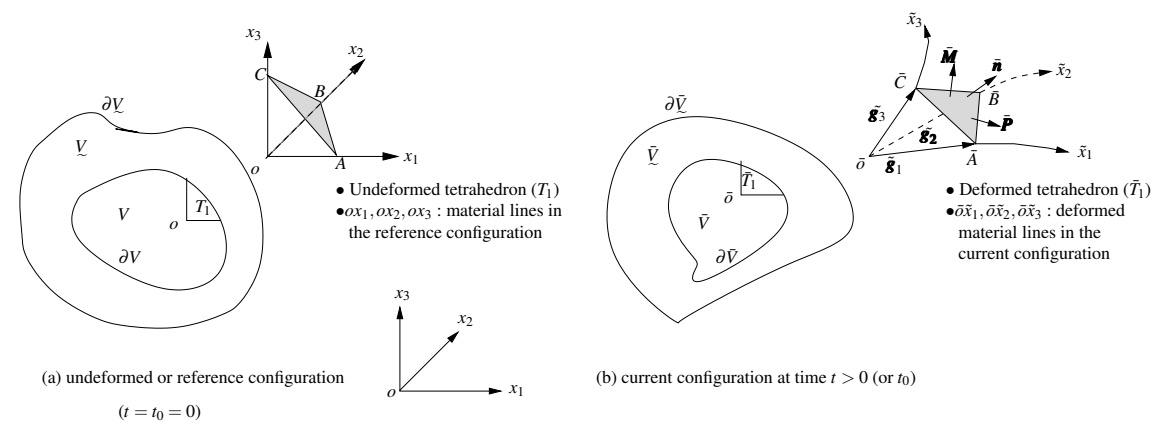

Figure 1: Reference and current configurations for a deforming volume of matter

\subsection{Covariant and contravariant bases}

The edges of the deformed tetrahedron $\bar{T}_{1}$ are covariant base vectors $\tilde{\boldsymbol{g}}_{i}$ that are tangent to the deformed material lines at $\bar{o}$. The faces of the tetrahedron are formed by the covariant base vectors $\tilde{\boldsymbol{g}}_{2}, \tilde{\boldsymbol{g}}_{3}, \tilde{\boldsymbol{g}}_{3}, \tilde{\boldsymbol{g}}_{1}$ and $\tilde{\boldsymbol{g}}_{1}, \tilde{\boldsymbol{g}}_{2}$. Following [59-61] we can define

$$
\tilde{\boldsymbol{g}}_{i}=\frac{\partial \bar{x}_{k}}{\partial x_{i}} \boldsymbol{e}_{k}
$$

$x_{i}$ and $\bar{x}_{k}$ being coordinates of a material point in the reference configuration and 
current configuration respectively. If $[J]$ is the Jacobian of deformation

$$
[J]=\frac{\partial\{\bar{x}\}}{\partial\{x\}} \quad \text { or } \quad J_{i j}=\frac{\partial \bar{x}_{i}}{\partial x_{j}}
$$

then the columns of $[J]$ are covariant base vectors $\tilde{\boldsymbol{g}}_{i}$. The contravariant basis are reciprocal to the covariant basis [59-61] are defined by the base vectors $\tilde{\boldsymbol{g}}^{i}$

$$
\tilde{\boldsymbol{g}}^{j}=\frac{\partial x_{j}}{\partial \bar{x}_{l}} \boldsymbol{e}_{l}
$$

We note that

$$
\tilde{\boldsymbol{g}}_{i} \cdot \tilde{\boldsymbol{g}}^{j}=\delta_{i j}
$$

Alternatively to (2.3) we can also define $\tilde{\boldsymbol{g}}^{i}$ as

$$
\begin{aligned}
\tilde{\boldsymbol{g}}^{1} & =\frac{\tilde{\boldsymbol{g}}_{2} \times \tilde{\boldsymbol{g}}_{3}}{\tilde{\boldsymbol{g}}_{1} \cdot\left(\tilde{\boldsymbol{g}}_{2} \times \tilde{\boldsymbol{g}}_{3}\right)} \\
\tilde{\boldsymbol{g}}^{2} & =\frac{\tilde{\boldsymbol{g}}_{3} \times \tilde{\boldsymbol{g}}_{1}}{\tilde{\boldsymbol{g}}_{2} \cdot\left(\tilde{\boldsymbol{g}}_{3} \times \tilde{\boldsymbol{g}}_{1}\right)} \\
\tilde{\boldsymbol{g}}^{3} & =\frac{\tilde{\boldsymbol{g}}_{1} \times \tilde{\boldsymbol{g}}_{2}}{\tilde{\boldsymbol{g}}_{3} \cdot\left(\tilde{\boldsymbol{g}}_{1} \times \tilde{\boldsymbol{g}}_{2}\right)}
\end{aligned}
$$

The volume of the parallelepiped framed by $\tilde{\boldsymbol{g}}_{i}$ in the current configuration is given by (same as denominators in (2.5))

$$
\bar{V}=\tilde{\boldsymbol{g}}_{1} \cdot\left(\tilde{\boldsymbol{g}}_{2} \times \tilde{\boldsymbol{g}}_{3}\right)=\tilde{\boldsymbol{g}}_{2} \cdot\left(\tilde{\boldsymbol{g}}_{3} \times \tilde{\boldsymbol{g}}_{1}\right)=\tilde{\boldsymbol{g}}_{3} \cdot\left(\tilde{\boldsymbol{g}}_{1} \times \tilde{\boldsymbol{g}}_{2}\right)
$$

We note that $\tilde{\boldsymbol{g}}^{i}$ in (2.3) as well as $\tilde{\boldsymbol{g}}^{j}$ in (2.5) satisfy (2.4). Thus definitions of $\tilde{\boldsymbol{g}}^{j}$ in (2.3) and (2.5) are exactly the same, as both definitions with (2.1) satisfy (2.4). We note that $\tilde{\boldsymbol{g}}^{1}, \tilde{\boldsymbol{g}}^{2}, \tilde{\boldsymbol{g}}^{3}$ are normal to the faces of the deformed tetrahedron formed by $\tilde{\boldsymbol{g}}_{2}$, $\tilde{\boldsymbol{g}}_{3} ; \tilde{\boldsymbol{g}}_{3}, \tilde{\boldsymbol{g}}_{1} ; \tilde{\boldsymbol{g}}_{1}, \tilde{\boldsymbol{g}}_{2}$ covariant base vectors. Covariant and contravariant directions are important in defining and choosing the correct measures of strains, stresses, moment intensities, etc. Under the action of $\overline{\boldsymbol{P}}$ and $\overline{\boldsymbol{M}}$ on surface $\bar{A} \bar{B} \bar{C}$ and the stress and moment intensities on the faces of the tetrahedron formed by $\tilde{\boldsymbol{g}}_{2}, \tilde{\boldsymbol{g}}_{3} ; \tilde{\boldsymbol{g}}_{3}, \tilde{\boldsymbol{g}}_{1}$; and $\tilde{\boldsymbol{g}}_{1}, \tilde{\boldsymbol{g}}_{2}$ base vectors, the tetrahedron $\bar{T}_{1}$ is in equilibrium.

\subsection{Definition of stress tensors}

\subsubsection{Contravariant Cauchy stress tensor}

The definition of the stresses on the non-oblique faces of the tetrahedron in the contravariant directions is the most natural way to define stress. Let ${\underset{\sim}{(0)}}^{\overline{o r}^{(0)}} \underbrace{\boldsymbol{\sigma}^{(0)}}$ be the 
contravariant stress tensor with components $\bar{\sigma}_{i j}^{(0)}$ or $\underset{\sigma_{i j}^{(0)}}{ }$ and dyads $\tilde{\boldsymbol{g}}_{i} \otimes \tilde{\boldsymbol{g}}_{j}$. Component $\bar{\sigma}_{11}^{(0)}$ or $\widetilde{\sigma}_{11}^{(0)}$ is in the $\tilde{\boldsymbol{g}}^{1}$ direction on a face of the tetrahedron with unit exterior normal $\tilde{\boldsymbol{g}}^{1}$, i.e., on the $\tilde{\boldsymbol{g}}^{1}$ face. Likewise $\bar{\sigma}_{12}^{(0)}$ or ${\underset{\sim}{12}}_{12}^{(0)}$ and $\bar{\sigma}_{31}^{(0)}$ or $\sigma_{31}^{(0)}$ act on the $\tilde{\boldsymbol{g}}^{1}$ and $\tilde{\boldsymbol{g}}^{3}$ faces in the $\tilde{\boldsymbol{g}}^{2}$ and $\tilde{\boldsymbol{g}}^{1}$ directions. Using the dyads $\tilde{\boldsymbol{g}}_{i} \otimes \tilde{\boldsymbol{g}}_{j}$ or contravariance law of transformation we can write

$$
\boldsymbol{\sigma}^{(0)}=\tilde{\boldsymbol{g}}_{i} \otimes \tilde{\boldsymbol{g}}_{j} \sigma_{i j}^{(0)}
$$

using (2.1) we can write

$$
\begin{aligned}
\boldsymbol{\sigma}^{(0)} & =\boldsymbol{e}_{i} \otimes \boldsymbol{e}_{j} \sigma_{i j}^{(0)} \\
\sigma_{i j}^{(0)} & =J_{i k}{\underset{\sim}{k l}}_{k l}^{(0)} J_{j l} \\
\text { or } \quad\left[\sigma^{(0)}\right]^{T} & =[J]\left[\underline{\sigma}^{(0)}\right][J]^{T}
\end{aligned}
$$

$\boldsymbol{\sigma}^{(0)}$ is the contravariant Cauchy stress tensor (Lagrangian) from which $\overline{\boldsymbol{\sigma}}^{(0)}$ can be easily obtained by replacing $[J]$ with $[\bar{J}]^{-1}$ and $\boldsymbol{\sigma}^{(0)}$ with $\overline{\boldsymbol{\sigma}}^{(0)}$ in (2.8). Since the dyads of $\boldsymbol{\sigma}^{(0)}$ or $\overline{\boldsymbol{\sigma}}^{(0)}$ are $\boldsymbol{e}_{i} \otimes \boldsymbol{e}_{j}$, the Cauchy principle holds between $\overline{\boldsymbol{P}}$ and $\overline{\boldsymbol{\sigma}}^{(0)}$, i.e.,

$$
\overline{\boldsymbol{P}}=\left(\overline{\boldsymbol{\sigma}}^{(0)}\right)^{T} \cdot \overline{\boldsymbol{n}}
$$

\subsubsection{Covariant Cauchy stress tensor}

Instead of using contravariant directions and stress components ${\underset{\sim}{\boldsymbol{\sigma}}}^{(0)}$ and covariant basis $\tilde{\boldsymbol{g}}_{i}$ we could use covariant stress components $\left(\underline{\sim}_{(0)}\right)_{i j}$ or $\left(\underline{\sim}_{(0)}\right)_{i j}$ and contravariant basis $\tilde{\boldsymbol{g}}^{i}$. Consideration of $\left(\underline{\sigma}_{(0)}\right)_{i j}$ of course will require a different deformed tetrahedron such that covariant vectors $\tilde{\boldsymbol{g}}_{i}$ are normal to its non-oblique faces. The adverse consequences of choosing this measure of stress for finite deformation are discussed in $[61,62]$. Here we proceed using this measure as an alternative to the contravariant stress measure. Using dyads $\tilde{\boldsymbol{g}}^{i} \otimes \tilde{\boldsymbol{g}}^{j}$ and components $\left({\underset{\sim}{(0)}}_{(0)}\right)_{i j}$ we can write

$$
\overline{\boldsymbol{\sigma}}_{(0)}=\tilde{\boldsymbol{g}}^{i} \otimes \tilde{\boldsymbol{g}}^{j}\left(\underline{\sigma}_{(0)}\right)_{i j}
$$

using (2.3) in (2.10) we can write 


$$
\begin{aligned}
\overline{\boldsymbol{\sigma}}_{(0)} & =\boldsymbol{e}_{i} \otimes \boldsymbol{e}_{j}\left(\overline{\boldsymbol{\sigma}}_{(0)}\right)_{i j} \\
\left(\overline{\boldsymbol{\sigma}}_{(0)}\right)_{i j} & =\bar{J}_{k i}\left({\underset{\sigma}{(0)}}_{(0)}\right)_{k l} \bar{J}_{l j} \\
\text { or } \quad\left[\overline{\boldsymbol{\sigma}}_{(0)}\right] & =[\bar{J}]^{T}\left[\underline{\sigma}_{(0)}\right][J]
\end{aligned}
$$

$\overline{\boldsymbol{\sigma}}_{(0)}$ is the covariant Cauchy stress tensor (Eulerian) from which $\boldsymbol{\sigma}_{(0)}$ can be obtained by replacing $[J]$ with $[J]^{-1}$ and $\overline{\boldsymbol{\sigma}}_{(0)}$ with $\boldsymbol{\sigma}^{(0)}$ in (2.11). Since the dyads of $\overline{\boldsymbol{\sigma}}_{(0)}$ are $\boldsymbol{e}_{i} \otimes \boldsymbol{e}_{j}$, the Cauchy principle holds between $\overline{\boldsymbol{P}}$ and $\overline{\boldsymbol{\sigma}}_{(0)}$, i.e.,

$$
\overline{\boldsymbol{P}}=\left(\overline{\boldsymbol{\sigma}}_{(0)}\right)^{T} \cdot \overline{\boldsymbol{n}}
$$

\section{Remark}

The Cauchy stress tensors $\boldsymbol{\sigma}^{(0)}$ or $\overline{\boldsymbol{\sigma}}^{(0)}$ and $\boldsymbol{\sigma}_{(0)}$ or $\overline{\boldsymbol{\sigma}}_{(0)}$ are nonsymmetric at this

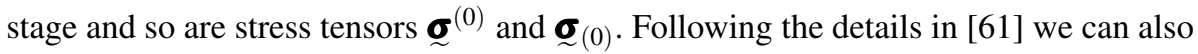
define Jaumann stress tensor ${ }^{(0)} \overline{\boldsymbol{\sigma}}^{J}$ using $\overline{\boldsymbol{\sigma}}^{(0)}$ and $\overline{\boldsymbol{\sigma}}_{(0)}$ stress measures.

\subsection{Definitions of moment tensors}

\subsubsection{Contravariant Cauchy moment tensor}

When the deformed tetrahedron with moment $\overline{\boldsymbol{M}}$ (per unit area) on its oblique face $\bar{A} \bar{B} \bar{C}$ is isolated from volume $\bar{V}$, its non-oblique face will have existence of moments (per unit area) on them. As in the case of stress, contravariant basis is the most natural way to define these. Let ${\underset{\sim}{\boldsymbol{m}}}^{(0)}$ or $\bar{\sim}^{(0)}$ be the contravariant moment tensors with components ${\underset{\sim}{(0)}}_{i j}^{(0)}$ or $\bar{m}_{i j}^{(0)}$ and dyads $\tilde{\boldsymbol{g}}_{i} \otimes \tilde{\boldsymbol{g}}_{j}$. Component ${\underset{\sim}{m}}_{11}^{(0)}$ or ${\stackrel{\sim}{\bar{m}_{11}}}_{11}^{(0)}$ is along $\tilde{\boldsymbol{g}}^{1}$ direction on a face of the tetrahedron with unit exterior normal $\tilde{\boldsymbol{g}}^{1}$, i.e., on $\tilde{\boldsymbol{g}}^{1}$ face. Likewise ${\underset{\sim}{12}}_{12}^{(0)}$ or $\bar{\sim}_{12}^{(0)}$ and ${\underset{\sim}{m 1}}_{31}^{(0)}$ or $\bar{\sim}_{31}^{(0)}$ act on $\tilde{\boldsymbol{g}}^{1}$ and $\tilde{\boldsymbol{g}}^{3}$ faces in the $\tilde{\boldsymbol{g}}^{2}$ and $\tilde{\boldsymbol{g}}^{1}$ directions. Using the dyads $\tilde{\boldsymbol{g}}_{i} \otimes \tilde{\boldsymbol{g}}_{j}$ or contravariance law of transformation we can write

$$
\boldsymbol{m}^{(0)}=\tilde{\boldsymbol{g}}_{i} \otimes \tilde{\boldsymbol{g}}_{j} m_{i j}^{(0)}
$$

Using (2.1) we can write 


$$
\begin{aligned}
\boldsymbol{m}^{(0)} & =\boldsymbol{e}_{i} \otimes \boldsymbol{e}_{j} m_{i j}^{(0)} \\
m_{i j}^{(0)} & =J_{i k} \underline{\sim}_{k l}^{(0)} J_{j l} \\
\text { or } \quad\left[m^{(0)}\right]^{T} & =[J]\left[m_{\sim}^{(0)}\right][J]^{T}
\end{aligned}
$$

$\boldsymbol{m}^{(0)}$ is contravariant Cauchy moment tensor (Lagrangian description) from which $\overline{\boldsymbol{m}}^{(0)}$ can be obtained by replacing $[J]$ with $[\bar{J}]^{-1}$ and $\boldsymbol{m}^{(0)}$ with $\overline{\boldsymbol{m}}^{(0)}$. Since the dyads of $\boldsymbol{m}^{(0)}$ or $\overline{\boldsymbol{m}}^{(0)}$ are $\boldsymbol{e}_{i} \otimes \boldsymbol{e}_{j}$, based on Koiter [12], the Cauchy principle is assumed to hold between $\overline{\boldsymbol{M}}$ and $\overline{\boldsymbol{m}}^{(0)}$, i.e.,

$$
\overline{\boldsymbol{M}}=\left(\overline{\boldsymbol{m}}^{(0)}\right)^{T} \cdot \overline{\boldsymbol{n}}
$$

We need to establish whether $\overline{\boldsymbol{m}}^{(0)}$ is symmetric or not, thus at this stage $\overline{\boldsymbol{m}}^{(0)}$ is not symmetric.

\subsubsection{Covariant Cauchy moment tensor}

Instead of using contravariant directions we could instead use covariant directions with moment tensor components $\left(\underset{\sim}{m_{(0)}}\right)_{i j}$ and contravariant basis with dyads $\tilde{\boldsymbol{g}}^{i} \otimes \tilde{\boldsymbol{g}}^{j}$. Consideration of $\left(\underset{\sim}{m_{(0)}}\right)_{i j}$ will of course require a different deformed tetrahedron such that covariant vectors $\tilde{\boldsymbol{g}}_{i}$ are normal to its non-oblique faces. The adverse consequences of choosing this measure are similar to those for the choice of $\left(\sigma_{(0)}\right)_{i j}$ for the stress measure. Using the dyads $\tilde{\boldsymbol{g}}^{i} \otimes \tilde{\boldsymbol{g}}^{j}$ with components $\left(\underline{\sim}_{(0)}\right)_{i j}$ we can write

$$
\overline{\boldsymbol{m}}_{(0)}=\tilde{\boldsymbol{g}}^{i} \otimes \tilde{\boldsymbol{g}}^{j}\left({\underset{\sim}{(0)}}_{(0)}\right)_{i j}
$$

Using (2.2) we can write

$$
\begin{aligned}
\overline{\boldsymbol{m}}_{(0)} & =\boldsymbol{e}_{i} \otimes \boldsymbol{e}_{j}\left(\bar{m}_{(0)}\right)_{i j} \\
\left(\bar{m}_{(0)}\right)_{i j} & =\bar{J}_{k i}\left(\underset{\sim}{m_{(0)}}\right)_{k l} \bar{J}_{l j} \\
\text { or } \quad\left[\bar{m}_{(0)}\right] & =[\bar{J}]^{T}\left[\underset{\sim}{\left.m_{(0)}\right]}\right][\bar{J}]
\end{aligned}
$$

$\overline{\boldsymbol{m}}_{(0)}$ is a covariant Cauchy moment tensor (Eulerian) from which $\boldsymbol{m}_{(0)}$ can be obtained by replacing $[J]$ with $[J]^{-1}$ and $\overline{\boldsymbol{m}}_{(0)}$ with $\boldsymbol{m}_{(0)}$. Following Koiter and since the dyads of $\overline{\boldsymbol{m}}_{(0)}$ are $\overline{\boldsymbol{e}}_{i} \otimes \overline{\boldsymbol{e}}_{j}$, the Cauchy principle holds between $\overline{\boldsymbol{M}}$ and $\overline{\boldsymbol{m}}_{(0)}$, i.e., 


$$
\overline{\boldsymbol{M}}=\left(\overline{\boldsymbol{m}}_{(0)}\right)^{T} \cdot \overline{\boldsymbol{n}}
$$

As in the case of the contravariant moment tensor, $\overline{\boldsymbol{m}}_{(0)}$ is also a non-symmetric Cauchy moment tensor in covariant basis unless established otherwise.

\subsection{First and second Piola-Kirchhoff stress and moment tensors}

In the Lagrangian description for deforming solid matter with finite deformation and finite strain, the Cauchy stress tensors and the Cauchy moment tensors in contraand covariant bases must be transformed into corresponding Piola-Kirchhoff tensors. Following [61] and assuming that $\boldsymbol{\sigma}_{(0)}$ and $\boldsymbol{\sigma}^{(0)}$ are nonsymmetric, we can derive the following relationship for the corresponding second Piola-Kirchhoff stress tensors $\boldsymbol{\sigma}_{[0]}$ and $\boldsymbol{\sigma}^{[0]}$, assuming the matter to be compressible.

$$
\begin{aligned}
& {\left[\sigma^{[0]}\right]=|J|[J]^{-1}\left[\sigma^{(0)}\right]^{T}\left[J^{T}\right]^{-1}} \\
& {\left[\sigma_{[0]}\right]=|J|[J]^{T}\left[\sigma_{(0)}\right]^{T}[J]}
\end{aligned}
$$

The first Piola-Kirchhoff stress tensor $\left(\boldsymbol{\sigma}^{*}\right)^{[0]}$ using contravariant Cauchy stress tensor $\boldsymbol{\sigma}^{(0)}$ can be expressed as

$$
\left[\left(\sigma^{*}\right)^{[0]}\right]^{T}=|J|\left[\sigma^{(0)}\right]^{T}\left[J^{T}\right]^{-1}
$$

Covariant $\left(\boldsymbol{\sigma}^{*}\right)_{[0]}$ due to covariant $\boldsymbol{\sigma}_{(0)}$ is of little use as covariant stress descriptions become non-physical, hence fail for finite deformation and finite strain [61,62].

Following the same approach as used for stress tensors we can also derive expressions for contravariant and covariant second Piola-Kirchhoff moment tensors using $\boldsymbol{m}^{(0)}$ and $\boldsymbol{m}_{(0)}$, contravariant and covariant Cauchy moment tensors, and the first PiolaKirchhoff contravariant moment tensor $\left(\boldsymbol{m}^{*}\right)^{[0]}$ using contravariant Cauchy moment tensor $\boldsymbol{m}^{(0)}$.

$$
\begin{aligned}
{\left[m^{[0]}\right] } & =|J|[J]^{-1}\left[m^{(0)}\right]^{T}\left[J^{T}\right]^{-1} \\
{\left[m_{[0]}\right] } & =|J|[J]^{T}\left[m_{(0)}\right]^{T}[J] \\
{\left[\left(m^{*}\right)^{[0]}\right]^{T} } & =|J|\left[m^{(0)}\right]^{T}\left[J^{T}\right]^{-1}
\end{aligned}
$$


We remark that at this stage $\boldsymbol{m}^{(0)}$ and $\boldsymbol{m}_{(0)}$ are nonsymmetric, hence $\boldsymbol{m}^{[0]}$ and $\boldsymbol{m}_{[0]}$ are nonsymmetric as well. $\left(\boldsymbol{m}^{*}\right)^{[0]}$ is always nonsymmetric regardless of $\boldsymbol{m}^{(0)}$. Just like $\left(\boldsymbol{\sigma}^{*}\right)_{[0]}$, the tensor $\left(\boldsymbol{m}^{*}\right)_{[0]}$ is of little use as they are both nonphysical $[61,62]$.

\subsection{Jacobian of deformation, internal rotations, their gradients and rates}

\subsubsection{Internal rotations, rotation matrix, and rotation gradients}

In finite deformation a tetrahedron in the undeformed configuration with its orthogonal edges deforms into one in which the edges are non-orthogonal covariant base vectors and the vectors normal to the faces of the deformed tetrahedron are contravariant non-orthogonal base vectors that are reciprocal to the covariant base vectors. The covariant and contravariant bases are fundamental in the measures of finite deformation, rotations, etc. Consider deformed coordinates $\overline{\boldsymbol{x}}$ of a material point in the current configuration with undeformed coordinates $\boldsymbol{x}$ in the reference configuration. Then

$$
\overline{\boldsymbol{x}}=\overline{\boldsymbol{x}}(\boldsymbol{x}) \quad \text { and } \quad \boldsymbol{x}=\boldsymbol{x}(\overline{\boldsymbol{x}})
$$

Let us define $\boldsymbol{J}$ as covariant Jacobian of deformation as its columns are covariant base vectors and $\overline{\boldsymbol{J}}$ as contravariant Jacobian of deformation whose rows are contravariant base vectors.

\section{Covariant $J$}

(a) Internal rotations and rotation matrix

Consider decomposition of the Jacobian of deformation $\boldsymbol{J}$ into symmetric and skew-symmetric tensors.

$$
\begin{gathered}
{[J]=\left[\frac{\partial\{\bar{x}\}}{\partial\{x\}}\right]=\left[{ }_{s} J\right]+\left[{ }_{a} J\right]} \\
{\left[{ }_{s} J\right]=\frac{1}{2}\left([J]+[J]^{T}\right)} \\
{\left[{ }_{a} J\right]=\frac{1}{2}\left([J]-[J]^{T}\right)}
\end{gathered}
$$

Let $\{\Theta\}=\left[\Theta_{x_{1}}, \Theta_{x_{2}}, \Theta_{x_{3}}\right]^{T}$ be the components of the rotations about covariant axes expressed as rotations about $o x_{1}, o x_{2}$, and $o x_{3}$ axes of the $x$-frame, then we can write 


$$
\left[{ }_{a} J\right]=\left[\begin{array}{ccc}
0 & \Theta_{x_{3}} & -\Theta_{x_{2}} \\
-\Theta_{x_{3}} & 0 & \Theta_{x_{1}} \\
\Theta_{x_{2}} & -\Theta_{x_{1}} & 0
\end{array}\right]
$$

in which

$$
\Theta_{x_{1}}=\frac{1}{2}\left(\frac{\partial u_{2}}{\partial x_{3}}-\frac{\partial u_{3}}{\partial x_{2}}\right) ; \quad \Theta_{x_{2}}=\frac{1}{2}\left(\frac{\partial u_{3}}{\partial x_{1}}-\frac{\partial u_{1}}{\partial x_{3}}\right) ; \quad \Theta_{x_{3}}=\frac{1}{2}\left(\frac{\partial u_{1}}{\partial x_{2}}-\frac{\partial u_{2}}{\partial x_{1}}\right)
$$

Alternatively we can also derive (2.30) as follows.

$$
\begin{aligned}
& \boldsymbol{\nabla} \times \boldsymbol{u}=\boldsymbol{e}_{i} \times \boldsymbol{e}_{j} \frac{\partial u_{j}}{\partial x_{i}}=\epsilon_{i j k} \boldsymbol{e}_{k} \frac{\partial u_{j}}{\partial x_{i}} \\
& \boldsymbol{\nabla} \times \boldsymbol{u}=\boldsymbol{e}_{1}\left(\frac{\partial u_{3}}{\partial x_{2}}-\frac{\partial u_{2}}{\partial x_{3}}\right)+\boldsymbol{e}_{2}\left(\frac{\partial u_{1}}{\partial x_{3}}-\frac{\partial u_{3}}{\partial x_{1}}\right)+\boldsymbol{e}_{3}\left(\frac{\partial u_{2}}{\partial x_{1}}-\frac{\partial u_{1}}{\partial x_{2}}\right) \\
& \boldsymbol{\nabla} \times \boldsymbol{u}=\boldsymbol{e}_{1}\left(-2 \Theta_{x_{1}}\right)+\boldsymbol{e}_{2}\left(-2 \Theta_{x_{2}}\right)+\boldsymbol{e}_{3}\left(-2 \Theta_{x_{3}}\right)
\end{aligned}
$$

$\epsilon_{i j k}$ is the permutation tensor.

The sign differences between (2.30) and (2.33) are due to clockwise and counterclockwise internal rotations and will only affect sign of $\overline{\boldsymbol{M}}$ term in the balance of angular momenta. If we use (2.30) as the definition of rotations then the term containing $\overline{\boldsymbol{M}}$ in the balance of angular momenta must have negative sign. If the rotations in (2.33) are defined as $\Theta_{x_{1}}, \Theta_{x_{2}}$, and $\Theta_{x_{3}}$ then the term containing $\overline{\boldsymbol{M}}$ in the balance of angular momenta must have positive sign. Regardless, the resulting equations and the following derivations are not affected. We note that decomposition in (2.26) enables explicit description of stretches and rotations contained in $\boldsymbol{J}$ due to deformation of solid matter. The stretch tensor and the rotation tensor can also be obtained using polar decomposition of $\boldsymbol{J}$ into right stretch tensor $\left(\boldsymbol{S}_{\boldsymbol{r}}\right)$ or left stretch tensor $\left(\boldsymbol{S}_{\boldsymbol{l}}\right)$ and pure rotation tensor $(\boldsymbol{R})$ [59-61].

$$
[J]=[R]\left[S_{r}\right]=\left[S_{l}\right][R]
$$

The stretch tensors $\boldsymbol{S}_{\boldsymbol{r}}$ and $\boldsymbol{S}_{\boldsymbol{l}}$ are symmetric and positive-definite and the rotation tensor $\boldsymbol{R}$ is orthogonal. Since $\boldsymbol{R}$ in (2.34) and $\boldsymbol{\Theta}$ in (2.30) are both obtained from the same deformation in $\boldsymbol{J}$, these contain details of the same internal rotation physics but in different forms. We make the following remarks. 
(i) $\boldsymbol{R}$ is rotation matrix, hence relates undeformed orthogonal frame to a new orthogonal rotated frame (due to deformation).

(ii) $\boldsymbol{\Theta}$ on the other hand contains rotation angles due to deformation about the axes of the $x$-frame.

(iii) We note that determination of $\boldsymbol{\Theta}$ from $\boldsymbol{R}$ or determination of $\boldsymbol{R}$ from $\boldsymbol{\Theta}$ is not necessary. Two different mathematical forms of rotation physics in $\boldsymbol{R}$ and $\boldsymbol{\Theta}$ is sufficient. However, we do remark that this process of obtaining $\boldsymbol{\Theta}$ from $\boldsymbol{R}$ or $\boldsymbol{R}$ from $\boldsymbol{\Theta}$ in general is not unique and may not even be possible without some approximation [63-65].

(iv) It suffices to note that internal rotations at a material point present in $\boldsymbol{J}$ can be expressed either in $\boldsymbol{R}$ or in $\boldsymbol{\Theta}$. Both forms contain mathematical description of same physics, hence either can be used as deemed suitable, but determination of $\boldsymbol{\Theta}$ from $\boldsymbol{R}$ or $\boldsymbol{R}$ from $\boldsymbol{\Theta}$ is not necessary.

(v) The internal rotation angles $\boldsymbol{\Theta}$ are present at every material point and are a result of deformation. Between two neighboring material points the variation of $\boldsymbol{\Theta}$ is perhaps small otherwise there may be permanent damage or separation between them. Regardless of the magnitude of $\boldsymbol{\Theta}$, these are strictly deterministic from ${ }_{a} \boldsymbol{J}$, $\boldsymbol{\nabla} \times \boldsymbol{u}$, or the polar decomposition.

(b) Internal rotation gradient tensor and its rates using $\mathbf{J}$

The covariant internal rotation tensor ${ }_{a} \boldsymbol{J}$ is a tensor of rank two, hence we can define

$$
{ }_{a} \boldsymbol{J}=\boldsymbol{e}_{i} \otimes \boldsymbol{e}_{j} \frac{1}{2}\left(\frac{\partial \bar{x}_{j}}{\partial x_{i}}-\frac{\partial \bar{x}_{i}}{\partial x_{j}}\right)
$$

Let ${ }^{\Theta} \boldsymbol{J}$ be the internal rotation gradient tensor, a tensor of rank three. Using (2.35) we can define

$$
{ }^{\Theta} \boldsymbol{J}=\boldsymbol{e}_{k} \otimes \boldsymbol{e}_{i} \otimes \boldsymbol{e}_{j} \frac{1}{2} \frac{\partial}{\partial x_{k}}\left(\frac{\partial \bar{x}_{j}}{\partial x_{i}}-\frac{\partial \bar{x}_{i}}{\partial x_{j}}\right)
$$

Alternatively (2.31) can be written as

$$
{ }_{a} \boldsymbol{J}=\epsilon_{i j l} \boldsymbol{e}_{l} \frac{1}{2}\left(\frac{\partial \bar{x}_{j}}{\partial x_{i}}-\frac{\partial \bar{x}_{i}}{\partial x_{j}}\right)
$$


and then

$$
{ }^{\Theta} \boldsymbol{J}=\boldsymbol{e}_{k} \otimes \boldsymbol{e}_{l} \epsilon_{i j l} \frac{1}{2} \frac{\partial}{\partial x_{k}}\left(\frac{\partial \bar{x}_{j}}{\partial x_{i}}-\frac{\partial \bar{x}_{i}}{\partial x_{j}}\right)
$$

In (2.35) the internal rotations ${ }_{a} \boldsymbol{J}$ are expressed as a tensor of rank one (i.e., $\Theta_{x_{1}}$, $\Theta_{x_{2}}, \Theta_{x_{3}}$ as a vector), hence its gradient ${ }^{\Theta} \boldsymbol{J}$ appears as a tensor of rank 2. The representation (2.37) is more appealing for matrix and vector representation given in the following. Let

$$
\{\Theta\}=\left[\Theta_{x_{1}}, \Theta_{x_{2}}, \Theta_{x_{3}}\right]^{T}
$$

Then we define rotation gradient tensor ${ }^{\Theta} \boldsymbol{J}$ and its decomposition into symmetric and skew-symmetric tensors ${ }_{s}^{\Theta} \boldsymbol{J}$ and ${ }_{a}^{\Theta} \boldsymbol{J}$.

$$
\begin{aligned}
& {\left[{ }^{\Theta} J\right]=\left[\frac{\partial\{\Theta\}}{\partial\{x\}}\right]=\left[{ }_{s}{ }_{s} J\right]+\left[{ }^{\Theta} J\right]} \\
& {\left[{ }_{s}{ }^{\Theta} J\right]=\frac{1}{2}\left(\left[{ }^{\Theta} J\right]+\left[{ }^{\Theta_{J}}\right]^{T}\right)} \\
& {\left[{ }_{a}{ }^{\Theta} J\right]=\frac{1}{2}\left(\left[{ }^{\Theta} J\right]-\left[{ }^{\Theta} J\right]^{T}\right)}
\end{aligned}
$$

We can also define the velocity gradients as

$$
\frac{\partial\{v\}}{\partial\{x\}}=[L]=[D]+[W]
$$

in which

$$
\begin{aligned}
{[D] } & =\frac{1}{2}\left([L]+[L]^{T}\right) \\
{[W] } & =\frac{1}{2}\left([L]-[L]^{T}\right)
\end{aligned}
$$

Likewise if ${ }^{t} \boldsymbol{\Theta}$ or $\dot{\boldsymbol{\Theta}}$ is the rotation rate then its gradients are given by

$$
\begin{gathered}
\frac{\partial\left\{{ }^{t} \Theta\right\}}{\partial\{x\}}=\left[{ }^{\Theta} L\right]=\left[{ }^{\Theta} D\right]+\left[{ }^{{ }}{ }_{W}\right] \\
{\left[{ }^{\Theta} D\right]=\frac{1}{2}\left(\left[{ }^{\Theta} L\right]+\left[{ }^{\Theta} L\right]{ }^{T}\right)}
\end{gathered}
$$




$$
\left[{ }^{\Theta} W\right]=\frac{1}{2}\left(\left[{ }^{\Theta} L\right]-\left[{ }^{\Theta} L\right]^{T}\right)
$$

\section{Remarks.}

(1) Symmetric rotation gradient tensor in (2.40) is a covariant measure in Lagrangian description. It describes symmetric part of the gradients in $x$-frame of rotations about covariant axes expressed about the axes of the $x$-frame.

(2) Just like Green's strain tensor (covariant measure) is conjugate with contravarian second Piola-Kirchhoff stress tensor derived using contravariant Cauchy stress tensor, ${ }_{s}^{\Theta} \boldsymbol{J}$ plays a significant role in conjugacy with the contravariant moment tensor $\left(\boldsymbol{m}^{*}\right)^{[0]}$.

(3) The covariant nature of this measure is intrinsic in its derivation due to $\boldsymbol{J}$, hence can not be changed. However, by replacing $\boldsymbol{J}$ with $\overline{\boldsymbol{J}}^{-1}$ these measures can be converted to Eulerian description.

\section{Contravariant $\overline{\boldsymbol{J}}$}

\section{(a) Internal rotations and rotation matrix}

Following the derivations for covariant measures, we can derive the following if we consider Jacobian of deformation $\overline{\boldsymbol{J}}$ in contravariant basis. Consider decomposition of $\overline{\boldsymbol{J}}$ into symmetric and skew-symmetric tensors.

$$
\begin{gathered}
{[\vec{J}]=\left[\frac{\partial\{\bar{x}\}}{\partial\{x\}}\right]=\left[{ }_{s} \vec{J}\right]+\left[{ }_{a} \vec{J}\right]} \\
{\left[{ }_{s} \vec{J}\right]=\frac{1}{2}\left([\vec{J}]+[\vec{J}]^{T}\right)} \\
{\left[{ }_{a} \vec{J}\right]=\frac{1}{2}\left([\vec{J}]-[\vec{J}]^{T}\right)}
\end{gathered}
$$

Let $\{\bar{\Theta}\}=\left[\bar{\Theta}_{x_{1}}, \bar{\Theta}_{x_{2}}, \bar{\Theta}_{x_{3}}\right]^{T}$ be the components of the rotations about covariant axes expressed as the rotations about $o x_{1}, o x_{2}$, and $o x_{3}$ axes of the $x$-frame, then we can write

$$
[a J]=\left[\begin{array}{ccc}
0 & \bar{\Theta}_{x_{3}} & -\bar{\Theta}_{x_{2}} \\
-\bar{\Theta}_{x_{3}} & 0 & \bar{\Theta}_{x_{1}} \\
\bar{\Theta}_{x_{2}} & -\bar{\Theta}_{x_{1}} & 0
\end{array}\right]
$$


in which

$$
\bar{\Theta}_{x_{1}}=\frac{1}{2}\left(\frac{\partial \bar{u}_{2}}{\partial \bar{x}_{3}}-\frac{\partial \bar{u}_{3}}{\partial \bar{x}_{2}}\right) ; \quad \bar{\Theta}_{x_{2}}=\frac{1}{2}\left(\frac{\partial \bar{u}_{3}}{\partial \bar{x}_{1}}-\frac{\partial \bar{u}_{1}}{\partial \bar{x}_{3}}\right) ; \quad \bar{\Theta}_{x_{3}}=\frac{1}{2}\left(\frac{\partial \bar{u}_{1}}{\partial \bar{x}_{2}}-\frac{\partial \bar{u}_{2}}{\partial \bar{x}_{1}}\right)
$$

Alternatively we can also derive (2.53) as follows.

$$
\begin{aligned}
& \overline{\boldsymbol{\nabla}} \times \overline{\boldsymbol{u}}=\boldsymbol{e}_{i} \times \boldsymbol{e}_{j} \frac{\partial \bar{u}_{j}}{\partial \bar{x}_{i}}=\epsilon_{i j k} \boldsymbol{e}_{k} \frac{\partial \bar{u}_{j}}{\partial \bar{x}_{i}} \\
& \overline{\boldsymbol{\nabla}} \times \overline{\boldsymbol{u}}=\boldsymbol{e}_{1}\left(\frac{\partial \bar{u}_{3}}{\partial \bar{x}_{2}}-\frac{\partial \bar{u}_{2}}{\partial \bar{x}_{3}}\right)+\boldsymbol{e}_{2}\left(\frac{\partial \bar{u}_{1}}{\partial \bar{x}_{3}}-\frac{\partial \bar{u}_{3}}{\partial \bar{x}_{1}}\right)+\boldsymbol{e}_{3}\left(\frac{\partial \bar{u}_{2}}{\partial \bar{x}_{1}}-\frac{\partial \bar{u}_{1}}{\partial \bar{x}_{2}}\right) \\
& \overline{\boldsymbol{\nabla}} \times \overline{\boldsymbol{u}}=\boldsymbol{e}_{1}\left(-2 \bar{\Theta}_{x_{1}}\right)+\boldsymbol{e}_{2}\left(-2 \bar{\Theta}_{x_{2}}\right)+\boldsymbol{e}_{3}\left(-2 \bar{\Theta}_{x_{3}}\right)
\end{aligned}
$$

The reason for the sign difference in (2.53) and (2.56) is exactly same as for covariant measures. We note that decomposition (2.49) enables explicit description of stretches (elongation per unit length and change in angles between the pair of orthogonal material lines in the undeformed configuration) and rotation tensor contained in $\overline{\boldsymbol{J}}$. The stretch tensors and the rotation tensor can also be obtained using polar decomposition of $\overline{\boldsymbol{J}}$ into right stretch tensor $\overline{\boldsymbol{S}}_{\boldsymbol{r}}$ or left stretch tensor $\overline{\boldsymbol{S}}_{\boldsymbol{l}}$ and rotation tensor $\overline{\boldsymbol{R}}$ [59-61].

$$
[\bar{J}]=[\bar{R}]\left[\bar{S}_{r}\right]=\left[\bar{S}_{l}\right][\bar{R}]
$$

The stretch tensors $\overline{\boldsymbol{S}}_{\boldsymbol{r}}$ and $\overline{\boldsymbol{S}}_{\boldsymbol{l}}$ are symmetric and positive-definite and the rotation tensor $\overline{\boldsymbol{R}}$ is orthogonal. Since $\overline{\boldsymbol{R}}$ in (2.57) and $\overline{\boldsymbol{\Theta}}$ in (2.53) are both obtained from the same deformation in $\overline{\boldsymbol{J}}$, these contain details of the same internal rotation physics but in different forms. We make the following remarks parallel to those for covariant measures.

(i) $\overline{\boldsymbol{R}}$ is rotation matrix due to deformation, hence relates two orthogonal frames.

(ii) $\overline{\boldsymbol{\Theta}}$ on the other hand contains rotation angles due to deformation about the axes of the $x$-frame due to rotations about contravariant axes.

(iii) We note that determination of $\overline{\boldsymbol{Q}}$ from $\overline{\boldsymbol{R}}$ or determination of $\overline{\boldsymbol{R}}$ from $\overline{\boldsymbol{\Theta}}$ is not necessary. Two different mathematical forms of rotation physics is sufficient in derivation of the conservation and balance laws. However, we do remark that this process of obtaining $\overline{\boldsymbol{\Theta}}$ from $\overline{\boldsymbol{R}}$ or $\overline{\boldsymbol{R}}$ from $\overline{\boldsymbol{\Theta}}$ in general is not unique and may not even be possible without some approximation [63-65]. 
(iv) It suffices to note that internal rotations at a material point present in $\overline{\boldsymbol{J}}$ can be expressed either in $\overline{\boldsymbol{R}}$ or in $\overline{\boldsymbol{\Theta}}$. Both forms contain mathematical description of same physics, hence either can be used as deemed suitable, but determination of $\overline{\boldsymbol{\Theta}}$ from $\overline{\boldsymbol{R}}$ or $\overline{\boldsymbol{R}}$ from $\overline{\boldsymbol{\Theta}}$ is not necessary.

(v) The internal rotation angles $\overline{\boldsymbol{\Theta}}$ are present at every material point and are a result of deformation. Between two neighboring material points the variation of $\overline{\boldsymbol{\Theta}}$ is perhaps small otherwise there may be permanent damage or separation between them. Regardless of the magnitude of $\overline{\mathbf{\Theta}}$, these are strictly deterministic from ${ }_{a} \overline{\boldsymbol{J}}$, $\overline{\boldsymbol{\nabla}} \times \overline{\boldsymbol{u}}$, or the polar decomposition.

\section{(b) Internal rotation gradient tensor using $\overline{\boldsymbol{J}}$}

The contravariant internal rotation tensor ${ }_{a} \overline{\boldsymbol{J}}$ is a tensor of rank two, hence we can define

$$
{ }_{a} \overline{\boldsymbol{J}}=\boldsymbol{e}_{i} \otimes \boldsymbol{e}_{j} \frac{1}{2}\left(\frac{\partial x_{j}}{\partial \bar{x}_{i}}-\frac{\partial x_{i}}{\partial \bar{x}_{j}}\right)
$$

Let ${ }^{\Theta} \overline{\boldsymbol{J}}$ be the internal rotation gradient tensor, a tensor of rank three. Using (2.58) we can define

$$
\Theta \overline{\boldsymbol{J}}=\boldsymbol{e}_{k} \otimes \boldsymbol{e}_{i} \otimes \boldsymbol{e}_{j} \frac{1}{2} \frac{\partial}{\partial \bar{x}_{k}}\left(\frac{\partial x_{j}}{\partial \bar{x}_{i}}-\frac{\partial x_{i}}{\partial \bar{x}_{j}}\right)
$$

Alternatively (2.58) can be written as

$$
{ }_{a} \overline{\boldsymbol{J}}=\epsilon_{i j l} \boldsymbol{e}_{l} \frac{1}{2}\left(\frac{\partial x_{j}}{\partial \bar{x}_{i}}-\frac{\partial x_{i}}{\partial \bar{x}_{j}}\right)
$$

and then

$$
\Theta \overline{\boldsymbol{J}}=\boldsymbol{e}_{k} \otimes \boldsymbol{e}_{l} \epsilon_{i j l} \frac{1}{2} \frac{\partial}{\partial \bar{x}_{k}}\left(\frac{\partial x_{j}}{\partial \bar{x}_{i}}-\frac{\partial x_{i}}{\partial \bar{x}_{j}}\right)
$$

In (2.59) the internal rotations ${ }_{a} \overline{\boldsymbol{J}}$ are expressed as a tensor of rank one (i.e., $\bar{\Theta}_{x_{1}}$, $\bar{\Theta}_{x_{2}}, \bar{\Theta}_{x_{3}}$ as a vector), hence its gradient ${ }^{\Theta} \overline{\boldsymbol{J}}$ appears as a tensor of rank 2 . The representation (2.60) is more appealing for matrix and vector representations given in the following. Let

$$
\{\bar{\Theta}\}=\left[\bar{\Theta}_{x_{1}}, \bar{\Theta}_{x_{2}}, \bar{\Theta}_{x_{3}}\right]^{T}
$$


Then we define rotation gradient tensor ${ }^{\Theta} \overline{\boldsymbol{J}}$ and its decomposition into symmetric and skew-symmetric tensors ${ }_{s}^{\Theta} \overline{\boldsymbol{J}}$ and ${ }_{a}^{\Theta} \overline{\boldsymbol{J}}$.

$$
\begin{aligned}
& {\left[{ }^{\Theta} \bar{J}\right]=\left[\frac{\partial\{\bar{\Theta}\}}{\partial\{\bar{x}\}}\right]=\left[{ }_{s}^{{ }_{s}} \bar{J}\right]+\left[{ }^{{ }_{a}} \bar{J}\right]} \\
& {\left[{ }^{\Theta} \bar{J}\right]=\frac{1}{2}\left(\left[{ }^{\Theta_{\bar{J}}}\right]+\left[{ }^{\Theta_{\bar{J}}}\right]^{T}\right)} \\
& {\left[\begin{array}{l}
{ }_{a} \\
a
\end{array}\right]=\frac{1}{2}\left(\left[{ }^{\Theta} \bar{J}\right]-\left[{ }^{\Theta} \bar{J}\right]^{T}\right)}
\end{aligned}
$$

\section{Remarks.}

(1) Symmetric rotation gradient tensor in (2.63) is a contravariant measure in Eulerian description. It describes symmetric part of the dradients of rotations about contravariant axes expressed about the axes of the $x$-frame.

(2) Since this measure is contravariant its work conjugate moment measure is expected to be covariant (see derivation of first law of thermodynamics).

(3) Contravariant nature of this measure is intrinsic in its derivation, hence can not be changed. However by replacing $\overline{\boldsymbol{J}}$ with $\boldsymbol{J}^{-1}$, these measures will become Lagrangian descriptions.

\subsubsection{Measure of finite strain}

For finite deformation Green's strain $\left(\boldsymbol{\varepsilon}_{[0]}\right)$ is a suitable choice in Lagrangian description for measure of finite strain. Following [59-61] we can write

$$
\left[\varepsilon_{[0]}\right]=\frac{1}{2}\left([J]^{T}[J]-[I]\right)
$$

Since

$$
[J]=[I]+\left[{ }^{d} J\right]=[I]+\left[\frac{\partial\{u\}}{\partial\{x\}}\right]
$$

$\left[\varepsilon_{[0]}\right]$ can be expressed in terms of $\left[{ }^{d} J\right]$.

$$
\left[\varepsilon_{[0]}\right]=\frac{1}{2}\left(\left[{ }^{d} \boldsymbol{J}\right]+\left[{ }^{d} \boldsymbol{J}\right]^{T}+\left[{ }^{d} \boldsymbol{J}\right]^{T}\left[{ }^{d} \boldsymbol{J}\right]\right)
$$




$$
\text { or } \quad\left(\varepsilon_{[0]}\right)_{i j}=\frac{1}{2}\left(\frac{\partial u_{j}}{\partial x_{i}}+\frac{\partial u_{i}}{\partial x_{j}}+\frac{\partial u_{i}}{\partial x_{k}} \frac{\partial u_{k}}{\partial x_{j}}\right)
$$

For infinitesimal deformation

$$
\begin{aligned}
{\left[\varepsilon_{[0]}\right] } & \simeq \frac{1}{2}\left(\left[{ }^{d} J\right]+\left[{ }^{d} J\right]^{T}\right) \\
\text { or } \quad\left(\varepsilon_{[0]}\right)_{i j} & \simeq \frac{1}{2}\left(\frac{\partial u_{j}}{\partial x_{i}}+\frac{\partial u_{i}}{\partial x_{j}}\right)
\end{aligned}
$$

This measure of strain is based on consideration of a length segment in reference configuration and its finite deformation in the current configuration. Such a measure is obviously not possible for the internal rotations as these do not exist in the reference configuration. Thus, in the finite deformation, finite strain internal polar non-classical continuum theories the explicit forms of internal rotations derived from $\boldsymbol{J}$ through $\boldsymbol{R}$ or ${ }_{a} \boldsymbol{J}$ remain the same as in the case of infinitesimal theory.

\subsubsection{Strain gradients and rotation gradients}

In subsequent sections of this paper we shall see that internal rotation gradients play an essential role in the constitutive theories for such solids. In [66], the author shows a relationship between the gradients of the rotations in terms of gradients of the strain tensor and the rotation tensor. Based on these and other similar works, it is argued and mostly accepted that the continuum theories that incorporate rotation gradients are same as those that are derived using strain gradients in the conservation and balance laws. The purpose of the material that follows is: (i) first to establish a relationship between the gradients of internal rotations and the gradients of the strain tensor (similar to [66]) and (ii) secondly, to demonstrate, using these relations, that the continuum theories based on rotation gradients and those based on strain gradients are in fact not the same. The resulting theories from the two approaches describe different physics. For simplicity, consider a two dimensional state of deformation in $x_{1} x_{2}$-plane. The displacement gradient tensor $\left[{ }^{d} J\right]$ (covariant basis, Lagrangian description) in this case is

$$
\left[{ }^{d} J\right]=\frac{\partial\left\{u_{1}, u_{2}\right\}}{\partial\left\{x_{1}, x_{2}\right\}}=\left[{ }_{s}^{d} J\right]+\left[{ }_{a}^{d} J\right]
$$


$\left[{ }_{s}^{d} J\right]$ and $\left[{ }_{a}^{d} J\right]$ being symmetric and antisymmetric tensors.

$$
\left[{ }_{a}^{d} J\right]=\left[\begin{array}{cc}
0 & \frac{1}{2}\left(\frac{\partial u_{1}}{\partial x_{2}}-\frac{\partial u_{2}}{\partial x_{1}}\right) \\
\frac{1}{2}\left(\frac{\partial u_{2}}{\partial x_{1}}-\frac{\partial u_{1}}{\partial x_{2}}\right) & 0
\end{array}\right]=\left[\begin{array}{cc}
0 & \Theta_{x_{3}} \\
-\Theta_{x_{3}} & 0
\end{array}\right]
$$

in which

$$
\Theta_{x_{3}}=\frac{1}{2}\left(\frac{\partial u_{1}}{\partial x_{2}}-\frac{\partial u_{2}}{\partial x_{1}}\right)=\Theta_{3}
$$

is the rotation about the $x_{3}$ axis. Gradients of $\Theta_{x_{3}}$ with respect to $x_{1}$ and $x_{2}$ are

$$
\begin{aligned}
& \Theta_{3,1}=\frac{1}{2}\left(\frac{\partial^{2} u_{1}}{\partial x_{1} \partial x_{2}}-\frac{\partial^{2} u_{2}}{\partial x_{1}^{2}}\right) \\
& \Theta_{3,2}=\frac{1}{2}\left(\frac{\partial^{2} u_{1}}{\partial x_{2}^{2}}-\frac{\partial^{2} u_{2}}{\partial x_{1} \partial x_{2}}\right)
\end{aligned}
$$

For small deformation, the strain measures are

$$
\begin{aligned}
\varepsilon_{11} & =\frac{\partial u_{1}}{\partial x_{1}} \\
\varepsilon_{22} & =\frac{\partial u_{2}}{\partial x_{2}} \\
\varepsilon_{12}=\varepsilon_{21} & =\frac{1}{2}\left(\frac{\partial u_{1}}{\partial x_{2}}+\frac{\partial u_{2}}{\partial x_{1}}\right)
\end{aligned}
$$

Substituting from (2.76) into (2.75) we can obtain

$$
\begin{aligned}
& \Theta_{3,1}=\frac{\partial \varepsilon_{11}}{\partial x_{2}}-\frac{\partial \varepsilon_{12}}{\partial x_{1}} \\
& \Theta_{3,2}=\frac{\partial \varepsilon_{12}}{\partial x_{2}}-\frac{\partial \varepsilon_{22}}{\partial x_{1}}
\end{aligned}
$$

In (2.77), the gradients $\Theta_{3,1}$ and $\Theta_{3,2}$ of rotation $\Theta_{x_{3}}$ are completely expressed in terms of the gradients of $\varepsilon_{11}$ and $\varepsilon_{22}$ with respect to $x_{2}$ and $x_{1}$ and $\varepsilon_{12}$ with respect to $x_{1}$ as well as $x_{2}$.

\section{Remarks}

(1) From (2.77) we note that gradients of $\Theta_{x_{3}}$ are functions of $\partial \varepsilon_{11} / \partial x_{2}, \partial \varepsilon_{22} / \partial x_{1}$, $\partial \varepsilon_{12} / \partial x_{1}$ and $\partial \varepsilon_{12} / \partial x_{2}$ but are not dependent on $\partial \varepsilon_{11} / \partial x_{1}$ and $\partial \varepsilon_{22} / \partial x_{2}$. This is expected due to the fact that $\partial \varepsilon_{11} / \partial x_{1}$ and $\partial \varepsilon_{22} / \partial x_{2}$ are gradients of the elongations per unit length in $x_{1}$ and $x_{2}$ directions, hence cannot possibly contribute to the gradients of rotations. 
(2) Considerations of $\Theta_{3,1}$ and $\Theta_{3,2}$ in the polar theory is identically equivalent to replacing these by the right side of the expressions in (2.77). As long as this condition is satisfied, the polar theory based on rotation gradients is the same as the polar theory based on strain gradients. We keep in mind that $\partial \varepsilon_{11} / \partial x_{1}$ and $\partial \varepsilon_{22} / \partial x_{2}$ are not part of the expressions of rotation gradients in (2.77).

(3) A polar theory based on strain gradients must consider $\varepsilon_{i j, k}$, i.e., gradients of all six strains with respect to $x_{1}, x_{2}$ and $x_{3}$. Thus, at the onset, it is clear that the strain gradient polar theory for the $2 \mathrm{D}$ case will also consider $\partial \varepsilon_{11} / \partial x_{1}$ and $\partial \varepsilon_{22} / \partial x_{2}$ in the derivation in addition to the other strain gradients that appear in (2.77). This undoubtedly brings in different physics than what is described by (2.77). If we consider three dimensional case (i.e., $\mathbb{R}^{3}$ ) then we would find that additionally $\partial \varepsilon_{33} / \partial x_{3}$ will appear in this strain gradient polar theory but will be absent in the definitions of the gradients of rotations.

(4) The consideration of the gradients of the internal rotations in the constitutive theories is supported by the entropy inequality (shown in subsequent section), while there is no rationale for the consideration of the strain gradients in the constitutive theories.

\section{Conservation and balance laws}

In this section we present conservation and balance laws. We assume the solid matter to be homogeneous, isotropic with finite deformation. The elastic solid matter can have dissipation mechanism as well as fading memory. Based on the assumption of thermodynamic equilibrium during deformation we consider: (i) conservation of mass, (ii) balance of linear momenta, (iii) balance of angular momenta, (iv) balance of moments of moments or couples, (v) first law of thermodynamics (energy equation), and (vi) second law of thermodynamics (entropy inequality).

The derivations are presented in Lagrangian description using contravariant first Piola-Kirchhoff stress tensor $\left(\boldsymbol{\sigma}^{*}\right)^{[0]}$ derived using contravariant Cauchy stress tensor and the contravariant first Piola-Kirchhoff moment tensor $\left(\boldsymbol{m}^{*}\right)^{[0]}$ derived using Contravariant Cauchy moment tensor. These choices of first Piola-Kirchhoff stress and moment measures are preferred in the derivation due to simplicity of the final equations in the balance laws. The final expressions can be transformed into any desired consistent measures. The reason for choosing contravariant stress and moment descriptions 
are well known $[61,62]$ as these conform to the physics of deformation. Due to Lagrangian description $\boldsymbol{J}$ is obviously the correct choice (compared to $\overline{\boldsymbol{J}}$ ) for Jacobian of deformation.

\subsection{Conservation of mass}

The derivation of the continuity equation from conservation of mass remains same as for non-polar continuum. Following [61] we can obtain the following continuity equation in Lagrangian description

$$
\rho_{0}(\boldsymbol{x})=|J| \rho(\boldsymbol{x}, t)
$$

$\rho_{0}(\boldsymbol{x})$ is the density in the reference configuration and $\rho(\boldsymbol{x}, t)$ is the Lagrangian description of the density of a material point at $\overline{\boldsymbol{x}}$ in the current configuration.

\subsection{Balance of linear momenta}

For a deforming volume of matter the rate of change of linear momenta must be equal to the sum of all other forces actying on it. This is Newton's second law applied to a volume of matter. This derivation is same as that in classical continuum theory. Thus, following [61] we can write (for finite deformation) the following using first and second Piola-Kirchhoff stress tensors $\left(\boldsymbol{\sigma}^{*}\right)^{[0]}$ and $\boldsymbol{\sigma}^{[0]}$ derived using contravariant Cauchy stress tensor.

$$
\left.\begin{array}{rlrl}
\rho_{0} \frac{D \boldsymbol{v}}{D t}-\rho_{0} \boldsymbol{F}^{b}-\boldsymbol{\nabla} \cdot\left(\boldsymbol{\sigma}^{*}\right)^{[0]} & =0 \\
\text { or } & \rho_{0} \frac{D\{v\}}{D t}-\rho_{0}\left\{F^{b}\right\}-\left[\left(\sigma^{*}\right)^{[0]}\right]^{T}\{\nabla\} & =0 \\
\text { or } & \rho_{0} \frac{D v_{i}}{D t}-\rho_{0} F_{i}^{b}-\frac{\partial\left(\sigma^{*}\right)_{j i}^{[0]}}{\partial x_{j}} & =0 \\
\text { or } & \rho_{0} \frac{D\{v\}}{D t}- & \rho_{0}\left\{F^{b}\right\}-\left[[J]\left[\sigma^{[0]}\right]^{T}\right]\{\nabla\} & =0
\end{array}\right\}
$$

In Lagrangian description $\frac{D}{D t}=\frac{\partial}{\partial t}$ holds. $\boldsymbol{v}=\boldsymbol{v}(\boldsymbol{x}, t)$ are velocities and $\boldsymbol{F}^{b}$ are body forces per unit mass. Equations (3.2) are momentum equations in $x_{1}, x_{2}$, and $x_{3}$ directions. 


\subsection{Balance of angular momenta}

The principle of balance of angular momenta for an internal polar non-classical continuum can be stated as follows: the time rate of change of total moment of momentum for an internal polar continuum is equal to the vector sum of the moments of external forces and the moments. Thus, due to surface stress $\overline{\boldsymbol{P}}$, surface moment $\overline{\boldsymbol{m}}$ (per unit area), body force $\overline{\boldsymbol{F}}^{b}$ (per unit mass), and the momentum $\bar{\rho} \overline{\boldsymbol{v}} d \overline{\boldsymbol{V}}$ for an elemental mass $\bar{\rho} d \bar{V}$ in the current configuration (using Eulerian description) we can write the following.

$$
\frac{D}{D t} \int_{\bar{V}(t)} \overline{\boldsymbol{x}} \times \bar{\rho} \overline{\boldsymbol{v}} d \bar{V}=\int_{\partial \bar{V}(t)}(\overline{\boldsymbol{x}} \times \overline{\boldsymbol{P}}-\overline{\boldsymbol{M}}) d \bar{A}+\int_{\bar{V}(t)} \overline{\boldsymbol{x}} \times \bar{\rho} \overline{\boldsymbol{F}}{ }^{b} d \bar{V}
$$

We consider each term in (3.3) individually.

$$
\begin{aligned}
& \frac{D}{D t} \int_{\bar{V}(t)} \overline{\boldsymbol{x}} \times \bar{\rho} \overline{\boldsymbol{v}} d \bar{V}=\frac{D}{D t} \int_{\bar{V}(t)} \epsilon_{i j k} \bar{x}_{i} \bar{v}_{j} \bar{\rho} d \bar{V} \\
& =\frac{D}{D t} \int_{V} \epsilon_{i j k} x_{i} v_{j} \rho_{0} d V \\
& =\int_{V} \rho_{0} \epsilon_{i j k}\left(\frac{D}{D t}\left(x_{i} v_{j}\right)\right) d V \\
& =\int_{V} \rho_{0} \epsilon_{i j k}\left(v_{i} v_{j}+x_{i} \frac{D v_{j}}{D t}\right) d V \\
& \int_{\partial \bar{V}(t)}(\overline{\boldsymbol{x}} \times \overline{\boldsymbol{P}}-\overline{\boldsymbol{M}}) d \bar{A}=\int_{\partial \bar{V}(t)}\left(\overline{\boldsymbol{x}} \times\left(\overline{\boldsymbol{\sigma}}^{(0)}\right)^{T} \cdot \overline{\boldsymbol{n}}-\left(\overline{\boldsymbol{m}}^{(0)}\right)^{T} \cdot \overline{\boldsymbol{n}}\right) d \bar{A} \\
& =\int_{\partial \bar{V}(t)} \overline{\boldsymbol{x}} \times\left(\overline{\boldsymbol{\sigma}}^{(0)}\right)^{T} \cdot \overline{\boldsymbol{n}} d \bar{A}-\int_{\partial \bar{V}(t)}\left(\overline{\boldsymbol{m}}^{(0)}\right)^{T} \cdot \overline{\boldsymbol{n}} d \bar{A} \\
& =\int_{\partial V} \boldsymbol{x} \times\left(\left(\boldsymbol{\sigma}^{*}\right)^{[0]}\right)^{T} \cdot \boldsymbol{n} d A-\int_{\partial V}\left(\left(\boldsymbol{m}^{*}\right)^{[0]}\right)^{T} \cdot \boldsymbol{n} d A \\
& =\int_{\partial V}\left(\epsilon_{i j k} x_{i}\left(\sigma^{*}\right)_{m j}^{[0]} n_{m}-\left(m^{*}\right)_{m k}^{[0]} n_{m}\right) d A
\end{aligned}
$$

Using divergence theorem 


$$
\begin{aligned}
\int_{\partial \bar{V}(t)}(\overline{\boldsymbol{x}} \times \overline{\boldsymbol{P}}-\overline{\boldsymbol{M}}) d \bar{A} & =\int_{V}\left(\epsilon_{i j k}\left(x_{i}\left(\boldsymbol{\sigma}^{*}\right)_{m j}^{[0]}\right)_{, m}-\left(\left(m^{*}\right)_{m k}^{[0]}\right)_{, m}\right) d V \\
& =\int_{V}\left(\epsilon_{i j k}\left(\delta_{i m}\left(\sigma^{*}\right)_{m j}^{[0]}+x_{i}\left(\left(\sigma^{*}\right)_{m j}^{[0]}\right)_{, m}\right)-\left(\left(m^{*}\right)_{m k}^{[0]}\right)_{, m}\right) d V \\
& =\int_{V}\left(\epsilon_{i j k}\left(\left(\sigma^{*}\right)_{i j}^{[0]}+x_{i}\left(\left(\sigma^{*}\right)_{m j}^{[0]}\right)_{, m}\right)-\left(\left(m^{*}\right)_{m k}^{[0]}\right)_{, m}\right) d V
\end{aligned}
$$

And

$$
\int_{\bar{V}(t)} \overline{\boldsymbol{x}} \times \bar{\rho} \overline{\boldsymbol{F}}^{b} d \bar{V}=\int_{\bar{V}(t)} \epsilon_{i j k} \bar{x}_{i} \bar{F}_{j}^{b} \bar{\rho} d \bar{V}=\int_{V} \epsilon_{i j k} x_{i} F_{j}^{b} \rho_{0} d V
$$

Substituting (3.4), (3.5), and (3.6) into (3.3)

$$
\begin{aligned}
& \int_{V} \rho_{0} \epsilon_{i j k}\left(v_{i} v_{j}+x_{i} \frac{D v_{j}}{D t}\right) d V= \\
& \quad \int_{V}\left(\epsilon_{i j k}\left(\left(\sigma^{*}\right)_{i j}^{[0]}+x_{i}\left(\left(\sigma^{*}\right)_{m j}^{[0]}\right)_{, m}\right)-\left(\left(m^{*}\right)_{m k}^{[0]}\right)_{, m}\right) d V+\int_{V} \epsilon_{i j k} x_{i} F_{j}^{b} \rho_{0} d V
\end{aligned}
$$

We note that

$$
\epsilon_{i j k} v_{i} v_{j}=0
$$

Hence, (3.7) reduces to

$$
\int_{V} \epsilon_{i j k} x_{i}\left(\rho_{0} \frac{D v_{j}}{D t}-\rho_{0} F_{j}^{b}-\left(\left(\sigma^{*}\right)_{m j}^{[0]}\right)_{, m}\right) d V+\int_{V}\left(-\left(\left(m^{*}\right)_{m k}^{[0]}\right)_{, m}+\epsilon_{i j k}\left(\sigma^{*}\right)_{i j}^{[0]}\right) d V=0
$$

Using balance of linear momenta (3.2) in (3.11) we obtain

$$
\int_{V}\left(-\left(\left(m^{*}\right)_{m k}^{[0]}\right)_{, m}+\epsilon_{i j k}\left(\sigma^{*}\right)_{i j}^{[0]}\right) d V=0
$$

Since the volume $V$ is arbitrary 


$$
\begin{array}{rlrl}
\left(\left(m^{*}\right)_{m k}^{[0]}\right)_{, m}-\epsilon_{i j k}\left(\boldsymbol{\sigma}^{*}\right)_{i j}^{[0]} & =0 \\
& \boldsymbol{\nabla} \cdot\left(\boldsymbol{m}^{*}\right)^{[0]}-\boldsymbol{\epsilon}:\left(\boldsymbol{\sigma}^{*}\right)^{[0]}=0 \\
\text { or } & & \\
\text { or } & {\left[\left(m^{*}\right)^{[0]}\right]^{T}\{\nabla\}-\epsilon:\left[\left(\sigma^{*}\right)^{[0]}\right]^{T}=0}
\end{array}
$$

\subsection{Balance of moment of moments}

In this derivation we have two choices. In the first we proceed with the fundamental statement in Eulerian description (neglecting inertial terms) [1] for moments of the moments given by

$$
\int_{\bar{V}} \overline{\boldsymbol{x}} \times\left(\epsilon: \overline{\boldsymbol{\sigma}}^{(0)}\right) d \bar{V}-\int_{\partial \bar{V}} \overline{\boldsymbol{x}} \times \overline{\boldsymbol{M}} d \bar{A}=0
$$

In (3.12) we transform all integrals for $\bar{V}$ and $\partial \bar{V}$ to $V$ and $\partial V$ and all measures to Lagrangian description to obtain the final results. In the second approach we proceed with final outcome of the balance of moments of moments in Eulerian description and then transform it to Lagrangian description, i.e., we begin with [1]

$$
\epsilon_{i j k} \bar{m}_{i j}^{(0)}=0
$$

from which we conclude that $\overline{\boldsymbol{m}}^{(0)}$ or its Lagrangian description $\boldsymbol{m}^{(0)}$ is symmetric. Using

$$
\begin{aligned}
& {\left[m^{[0]}\right]^{T}=|J|[J]^{-1}\left[m^{(0)}\right]^{T}\left[J^{T}\right]^{-1} } \\
\text { and } \quad & {\left[\left(m^{*}\right)^{[0]}\right]^{T}=|J|\left[m^{(0)}\right]^{T}\left[J^{T}\right]^{-1} }
\end{aligned}
$$

we conclude that if $\boldsymbol{m}^{(0)}$ is symmetric, then $\boldsymbol{m}^{[0]}$, the second Piola-Kirchhoff moment tensor, is symmetric as well, however $\left(\boldsymbol{m}^{*}\right)^{[0]}$ is not symmetric.

\subsection{First law of thermodynamics}

The sum of work and heat added to a deforming volum,e of matter must result in the increase of the energy of the system. Expressing this as a rate equation in Eulerian description we can write [61]

$$
\frac{D \bar{E}_{t}}{D t}=\frac{D \bar{Q}}{D t}+\frac{D \bar{W}}{D t}
$$


$\bar{E}_{t}, \bar{Q}$, and $\bar{W}$ are total energy, heat added, and work done. These can be written as

$$
\begin{gathered}
\frac{D \bar{E}_{t}}{D t}=\frac{D}{D t} \int_{\bar{V}(t)} \bar{\rho}\left(\bar{e}+\frac{1}{2} \overline{\boldsymbol{v}} \cdot \overline{\boldsymbol{v}}-\overline{\boldsymbol{F}}^{b} \cdot \overline{\boldsymbol{u}}\right) d \bar{V} \\
\frac{D \bar{Q}}{D t}=-\int_{\partial \bar{V}(t)} \overline{\boldsymbol{q}} \cdot \overline{\boldsymbol{n}} d \bar{A} \\
\frac{D \bar{W}}{D t}=\int_{\partial \bar{V}(t)}\left(\overline{\boldsymbol{P}} \cdot \overline{\boldsymbol{v}}+\overline{\boldsymbol{M}} \cdot{ }^{t} \overline{\mathbf{\Theta}}\right) d \bar{A}
\end{gathered}
$$

where $\bar{e}$ is specific internal energy, $\overline{\boldsymbol{F}}^{b}$ is body force vector, $\overline{\boldsymbol{q}}$ is rate of heat. In (3.17) we have neglected rotary intertia. This is consistent with the assumptions used in the conservation law in subsection 3.1. We expand integrals in (3.17)-(3.19). Following [61] we can show the following.

$$
\frac{D}{D t} \int_{\bar{V}(t)} \bar{\rho}\left(\bar{e}+\frac{1}{2} \overline{\boldsymbol{v}} \cdot \overline{\boldsymbol{v}}-\overline{\boldsymbol{F}}^{b} \cdot \overline{\boldsymbol{u}}\right) d \bar{V}=\int_{V}\left(\rho_{0} \frac{D e}{D t}+\rho_{0} \boldsymbol{v} \cdot \frac{D \boldsymbol{v}}{D t}-\boldsymbol{F}^{b} \cdot \boldsymbol{v}\right) d V
$$

Using

$$
\begin{array}{r}
\overline{\boldsymbol{q}} \cdot \overline{\boldsymbol{n}} d \bar{A}=\boldsymbol{q} \cdot \boldsymbol{n} d A ; \quad \bar{\rho} d \bar{V}=\rho_{0} d V ; \quad d \bar{V}=|J| d V \\
-\int_{\partial \bar{V}(t)} \overline{\boldsymbol{q}} \cdot \overline{\boldsymbol{n}} d \bar{A}=-\int_{\partial V} \boldsymbol{q} \cdot \boldsymbol{n} d A=-\int_{V} \boldsymbol{\nabla} \cdot \boldsymbol{q} d V ; \quad \text { Divergence theorem }
\end{array}
$$

Using contravariant Cauchy stress tensor and contravariant Cauchy moment tensor and first Piola-Kirchhoff stress and moment tensors we can derive the following [61].

$$
\begin{aligned}
\overline{\boldsymbol{P}} \cdot \overline{\boldsymbol{v}} d \bar{A} & =\left(\boldsymbol{v} \cdot\left(\left(\boldsymbol{\sigma}^{*}\right)^{[0]}\right)^{T}\right) \cdot \boldsymbol{n} d A=\left(\boldsymbol{v} \cdot\left(\left(\boldsymbol{\sigma}^{*}\right)^{[0]}\right)^{T}\right) \cdot d \boldsymbol{A} \\
\overline{\boldsymbol{M}} \cdot{ }^{t} \overline{\boldsymbol{\Theta}} d \bar{A} & =\left({ }^{t} \boldsymbol{\Theta} \cdot\left(\left(\boldsymbol{m}^{*}\right)^{[0]}\right)^{T}\right) \cdot \boldsymbol{n} d A=\left({ }^{t} \boldsymbol{\Theta} \cdot\left(\left(\boldsymbol{m}^{*}\right)^{[0]}\right)^{T}\right) \cdot d \boldsymbol{A}
\end{aligned}
$$

Thus we can write the following for (3.16). 


$$
\begin{aligned}
\int_{V}\left(\rho_{0} \frac{D e}{D t}+\rho_{0} \boldsymbol{v} \cdot \frac{D \boldsymbol{v}}{D t}-\boldsymbol{F}^{b} \cdot \boldsymbol{v}\right) d V= \\
\quad-\int_{V} \boldsymbol{\nabla} \cdot \boldsymbol{q} d V+\int_{\partial V}\left(\boldsymbol{v} \cdot\left(\left(\boldsymbol{\sigma}^{*}\right)^{[0]}\right)^{T}\right) \cdot d \boldsymbol{A}+\int_{\partial V}\left({ }^{t} \boldsymbol{\Theta} \cdot\left(\left(\boldsymbol{m}^{*}\right)^{[0]}\right)^{T}\right) \cdot d \boldsymbol{A}
\end{aligned}
$$

Using divergence theorem for integrals over $\partial V$

$$
\begin{aligned}
\int_{V} & \left(\rho_{0} \frac{D e}{D t}+\rho_{0} \boldsymbol{v} \cdot \frac{D \boldsymbol{v}}{D t}-\boldsymbol{F}^{b} \cdot \boldsymbol{v}\right) d V \\
& -\int_{V} \boldsymbol{\nabla} \cdot \boldsymbol{q} d V+\int_{V} \boldsymbol{\nabla} \cdot\left(\boldsymbol{v} \cdot\left(\left(\boldsymbol{\sigma}^{*}\right)^{[0]}\right)^{T}\right) d V+\int_{V} \boldsymbol{\nabla} \cdot\left({ }^{t} \boldsymbol{\Theta} \cdot\left(\left(\boldsymbol{m}^{*}\right)^{[0]}\right)^{T}\right) d V
\end{aligned}
$$

Following [61] we can also show that

$$
\begin{aligned}
\boldsymbol{\nabla} \cdot\left(\boldsymbol{v} \cdot\left(\left(\boldsymbol{\sigma}^{*}\right)^{[0]}\right)^{T}\right) & =\boldsymbol{v} \cdot\left(\boldsymbol{\nabla} \cdot\left(\boldsymbol{\sigma}^{*}\right)^{[0]}\right)+\left(\boldsymbol{\sigma}^{*}\right)_{j i}^{[0]} \frac{\partial v_{i}}{\partial x_{j}} \\
\text { and } \quad \boldsymbol{\nabla} \cdot\left({ }^{t} \boldsymbol{Q} \cdot\left(\left(\boldsymbol{m}^{*}\right)^{[0]}\right)^{T}\right) & ={ }^{t} \boldsymbol{Q} \cdot\left(\boldsymbol{\nabla} \cdot\left(\boldsymbol{m}^{*}\right)^{[0]}\right)+\left(m^{*}\right)_{j i}^{[0]} \frac{\partial^{t} \Theta_{x_{i}}}{\partial x_{j}}
\end{aligned}
$$

Substituting (3.27) and (3.28) into (3.26)

$$
\begin{aligned}
& \int_{V}\left(\rho_{0} \frac{D e}{D t}+\rho_{0} \boldsymbol{v} \cdot \frac{D \boldsymbol{v}}{D t}-\boldsymbol{F}^{b} \cdot \boldsymbol{v}\right) d V \\
= & -\int_{V} \boldsymbol{\nabla} \cdot \boldsymbol{q} d V+\int_{V}\left(\boldsymbol{v} \cdot\left(\boldsymbol{\nabla} \cdot\left(\boldsymbol{\sigma}^{*}\right)^{[0]}\right)+\left(\boldsymbol{\sigma}^{*}\right)_{j i}^{[0]} \frac{\partial v_{i}}{\partial x_{j}}+{ }^{t} \boldsymbol{\Theta} \cdot\left(\boldsymbol{\nabla} \cdot\left(\boldsymbol{m}^{*}\right)^{[0]}\right)+\left(m^{*}\right)_{j i}^{[0]} \frac{\partial^{t} \Theta_{x_{i}}}{\partial x_{j}}\right) d V
\end{aligned}
$$

Moving all terms to the left side and regrouping

$$
\begin{aligned}
& \int_{V} \rho_{0} \boldsymbol{v} \cdot\left(\frac{D \boldsymbol{v}}{D t}-\boldsymbol{F}^{b}-\boldsymbol{\nabla} \cdot\left(\boldsymbol{\sigma}^{*}\right)^{[0]}\right) d V \\
& +\int_{V}\left(\rho_{0} \frac{D e}{D t}+\boldsymbol{\nabla} \cdot \boldsymbol{q}-\left(\sigma^{*}\right)_{j i}^{[0]} \frac{\partial v_{i}}{\partial x_{j}}-\left(m^{*}\right)_{j i}^{[0]} \frac{\partial^{t} \Theta_{x_{i}}}{\partial x_{j}}-{ }^{t} \boldsymbol{\Theta} \cdot\left(\boldsymbol{\nabla} \cdot\left(\boldsymbol{m}^{*}\right)^{[0]}\right)\right) d V=0
\end{aligned}
$$


Using (3.2) (balance of linear momenta), (3.30) reduces to

$$
\int_{V}\left(\rho_{0} \frac{D e}{D t}+\boldsymbol{\nabla} \cdot \boldsymbol{q}-\left(\sigma^{*}\right)_{j i}^{[0]} \frac{\partial v_{i}}{\partial x_{j}}-\left(m^{*}\right)_{j i}^{[0]} \frac{\partial^{t} \Theta_{x_{i}}}{\partial x_{j}}-{ }^{t} \boldsymbol{\Theta} \cdot\left(\boldsymbol{\nabla} \cdot\left(\boldsymbol{m}^{*}\right)^{[0]}\right)\right) d V=0
$$

Since volume $V$ is arbitrary, we have

$$
\rho_{0} \frac{D e}{D t}+\boldsymbol{\nabla} \cdot \boldsymbol{q}-\left(\sigma^{*}\right)_{j i}^{[0]} \frac{\partial v_{i}}{\partial x_{j}}-\left(m^{*}\right)_{j i}^{[0]} \frac{\partial^{t} \Theta_{x_{i}}}{\partial x_{j}}-{ }^{t} \boldsymbol{\Theta} \cdot\left(\boldsymbol{\nabla} \cdot\left(\boldsymbol{m}^{*}\right)^{[0]}\right)=0
$$

We note in the ${ }^{t} \boldsymbol{\Theta} \cdot\left(\boldsymbol{\nabla} \cdot\left(\boldsymbol{m}^{*}\right)^{[0]}\right)$ or $\dot{\boldsymbol{\Theta}} \cdot\left(\boldsymbol{\nabla} \cdot\left(\boldsymbol{m}^{*}\right)^{[0]}\right)$ terms, $\boldsymbol{\nabla} \cdot\left(\boldsymbol{m}^{*}\right)^{[0]}$ can be substituted from (3.11) thereby eliminating gradients of $\left(\boldsymbol{m}^{*}\right)^{[0]}$ but introducing $\left(\boldsymbol{\sigma}^{*}\right)^{[0]}$.

\subsection{Second law of thermodynamics}

If $\bar{\eta}$ is entropy density in volume $\bar{V}(t), \bar{h}$ is the entropy flux between $\bar{V}(t)$ and the volume of matter surrounding it, and $\bar{s}$ is the source of entropy in $\bar{V}(t)$ due to noncontacting bodies then the rate of increase of entropy in volume $\bar{V}(t)$ is at least equal to that supplied to $\bar{V}(t)$ from all contacting and non-contacting sources [61]. Thus

$$
\frac{D}{D t} \int_{\bar{V}(t)} \bar{\eta} \bar{\rho} d \bar{V} \geq \int_{\partial \bar{V}(t)} \bar{h} d \bar{A}+\int_{\bar{V}(t)} \bar{s} \bar{\rho} d \bar{V}
$$

Using Cauchy's postulate for $\bar{h}$, i.e.,

$$
\begin{gathered}
\bar{h}=-\overline{\boldsymbol{\Psi}} \cdot \overline{\boldsymbol{n}} \\
\frac{D}{D t} \int_{\bar{V}(t)} \bar{\eta} \bar{\rho} d \bar{V} \geq-\int_{\partial \bar{V}(t)} \overline{\boldsymbol{\Psi}} \cdot \overline{\boldsymbol{n}} d \bar{A}+\int_{\bar{V}(t)} \bar{s} \bar{\rho} d \bar{V}
\end{gathered}
$$

We need to transform (3.35) into Lagrangian description. This can be done using

$$
\overline{\boldsymbol{\Psi}} \cdot \overline{\boldsymbol{n}} d \bar{A}=\boldsymbol{\Psi} \cdot \boldsymbol{n} d A ; \quad \bar{\rho} d \bar{V}=\rho_{0} d V ; \quad d \bar{V}=|J| d V
$$

Using (3.36) in (3.35)

$$
\frac{D}{D t} \int_{V} \eta \rho_{0} d V \geq-\int_{\partial V} \boldsymbol{\Psi} \cdot \boldsymbol{n} d A+\int_{V} s \rho_{0} d V
$$


Using Gauss's divergence theorem for the term over $\partial V$ gives (noting that $\boldsymbol{\Psi}$ is a tensor of rank one)

$$
\frac{D}{D t} \int_{V} \eta \rho_{0} d V \geq-\int_{V} \boldsymbol{\nabla} \cdot \boldsymbol{\Psi} d V+\int_{V} s \rho_{0} d V
$$

or

$$
\int_{V}\left(\rho_{0} \frac{D \eta}{D t}+\boldsymbol{\nabla} \cdot \boldsymbol{\Psi}-s \rho_{0}\right) d V \geq 0
$$

Since volume $V$ is arbitrary

$$
\rho_{0} \frac{D \eta}{D t}+\boldsymbol{\nabla} \cdot \boldsymbol{\Psi}-s \rho_{0} \geq 0
$$

Equation (3.40) is entropy inequality and is the most fundamental form resulting from the second law of thermodynamics. A more useful form can be derived if we assume

$$
\boldsymbol{\Psi}=\frac{\boldsymbol{q}}{\theta} ; \quad s=\frac{r}{\theta}
$$

where $\theta$ is absolute temperature, $\boldsymbol{q}$ is heat vector, and $r$ is a suitable potential, then

$$
\boldsymbol{\nabla} \cdot \boldsymbol{\Psi}=\Psi_{i, i}=\frac{q_{i, i}}{\boldsymbol{\theta}}-\frac{q_{i} \theta_{, i}}{\theta^{2}}=\frac{q_{i, i}}{\theta}-\frac{q_{i} g_{i}}{\theta^{2}}=\frac{\boldsymbol{\nabla} \cdot \boldsymbol{q}}{\boldsymbol{\theta}}-\frac{\boldsymbol{q} \cdot \boldsymbol{g}}{\theta^{2}}
$$

in which $\{g\}=\{\nabla \theta\}$ is the temperature gradient. Substituting for (3.41) and (3.42) into (3.40) and multiplying throughout by $\theta$

$$
\rho_{0} \frac{D \eta}{D t}+\left(\boldsymbol{\nabla} \cdot \boldsymbol{q}-\rho_{0} r\right)-\frac{\boldsymbol{q} \cdot \boldsymbol{g}}{\theta} \geq 0
$$

From energy equation (3.32) (after inserting $\rho_{0} r$ term) in contravariant basis

$$
\boldsymbol{\nabla} \cdot \boldsymbol{q}-\rho_{0} r=-\rho_{0} \frac{D e}{D t}+\left(\sigma^{*}\right)_{j i}^{[0]} \frac{\partial v_{i}}{\partial x_{j}}+\left(m^{*}\right)_{j i}^{[0]} \frac{\partial^{t} \Theta_{x_{i}}}{\partial x_{j}}+{ }^{t} \boldsymbol{\Theta} \cdot\left(\boldsymbol{\nabla} \cdot\left(\boldsymbol{m}^{*}\right)^{[0]}\right)
$$

Substituting from (3.44) into (3.43)

$$
\rho_{0} \frac{D \eta}{D t}+\left(-\rho_{0} \frac{D e}{D t}+\left(\sigma^{*}\right)_{j i}^{[0]} \frac{\partial v_{i}}{\partial x_{j}}+\left(m^{*}\right)_{j i}^{[0]} \frac{\partial^{t} \Theta_{x_{i}}}{\partial x_{j}}+{ }^{t} \boldsymbol{\Theta} \cdot\left(\boldsymbol{\nabla} \cdot\left(\boldsymbol{m}^{*}\right)^{[0]}\right)\right)-\frac{\boldsymbol{q} \cdot \boldsymbol{8}}{\theta} \geq 0
$$




$$
\rho_{0}\left(\frac{D e}{D t}-\theta \frac{D \eta}{D t}\right)+\frac{\boldsymbol{q} \cdot \boldsymbol{g}}{\theta}-\left(\sigma^{*}\right)_{j i}^{[0]} \frac{\partial v_{i}}{\partial x_{j}}-\left(m^{*}\right)_{j i}^{[0]} \frac{\partial^{t} \Theta_{x_{i}}}{\partial x_{j}}-{ }^{t} \boldsymbol{\Theta} \cdot\left(\boldsymbol{\nabla} \cdot\left(\boldsymbol{m}^{*}\right)^{[0]}\right) \leq 0
$$

Let $\Phi$ be the Helmholtz free energy density defined by

$$
\begin{gathered}
\Phi=e-\eta \theta \\
\therefore \quad \frac{D e}{D t}-\theta \frac{D \eta}{D t}=\frac{D \Phi}{D t}+\eta \frac{D \theta}{D t}
\end{gathered}
$$

Substituting from (3.48) into (3.46) we obtain

$$
\rho_{0}\left(\frac{D \Phi}{D t}+\eta \frac{D \theta}{D t}\right)+\frac{\boldsymbol{q} \cdot \boldsymbol{g}}{\theta}-\left(\sigma^{*}\right)_{j i}^{[0]} \frac{\partial v_{i}}{\partial x_{j}}-\left(m^{*}\right)_{j i}^{[0]} \frac{\partial^{t} \Theta_{x_{i}}}{\partial x_{j}}-{ }^{t} \boldsymbol{\Theta} \cdot\left(\boldsymbol{\nabla} \cdot\left(\boldsymbol{m}^{*}\right)^{[0]}\right) \leq 0
$$

We note that

$$
\left(\sigma^{*}\right)_{j i}^{[0]} \frac{\partial v_{i}}{\partial x_{j}}=\operatorname{tr}\left(\left[\left(\sigma^{*}\right)^{[0]}\right]^{T}[\dot{J}]^{T}\right)
$$

and

$$
\left(m^{*}\right)_{j i}^{[0]} \frac{\partial^{t} \Theta_{x_{i}}}{\partial x_{j}}=\operatorname{tr}\left(\left[\left(m^{*}\right)^{[0]}\right]^{T}\left[{ }^{\Theta} j^{T}\right]^{T}\right)
$$

\subsection{Stress and moment decompositions, conjugate pairs, and the final form of the conservation and balance laws}

The energy equation (3.32) and the entropy inequality (3.49) in these forms are not suitable for deciding on rate of work conjugate pairs that are essential for constitutive theories. In order to determine these we must consider decomposition of stress tensor, moment tensor, and the quantities associated with these in the energy equation and the entropy inequality. Since $\left(\boldsymbol{\sigma}^{*}\right)^{[0]}$ and $\left(\boldsymbol{m}^{*}\right)^{[0]}$ both are non-symmetric we consider their decomposition into symmetric and skew-symmetric tensors.

$$
\begin{aligned}
& \left(\boldsymbol{\sigma}^{*}\right)^{[0]}={ }_{s}\left(\boldsymbol{\sigma}^{*}\right)^{[0]}+{ }_{a}\left(\boldsymbol{\sigma}^{*}\right)^{[0]} \\
& \left(\boldsymbol{m}^{*}\right)^{[0]}={ }_{s}\left(\boldsymbol{m}^{*}\right)^{[0]}+{ }_{a}\left(\boldsymbol{m}^{*}\right)^{[0]}
\end{aligned}
$$


Hence

$$
\begin{aligned}
& \boldsymbol{\epsilon}:\left(\boldsymbol{\sigma}^{*}\right)^{[0]}=\boldsymbol{\epsilon}:\left({ }_{s}\left(\boldsymbol{\sigma}^{*}\right)^{[0]}+{ }_{a}\left(\boldsymbol{\sigma}^{*}\right)^{[0]}\right)=\boldsymbol{\epsilon}:{ }_{a}\left(\boldsymbol{\sigma}^{*}\right)^{[0]} \\
& \text { Since } \quad \boldsymbol{\epsilon}:{ }_{s}\left(\boldsymbol{\sigma}^{*}\right)^{[0]}=0 \\
& \operatorname{tr}\left(\left[\left(\sigma^{*}\right)^{[0]}\right]^{T}[\dot{J}]^{T}\right)=\operatorname{tr}\left(\left[\left(\sigma^{*}\right)^{[0]}\right][\dot{J}]\right) \\
& =\operatorname{tr}\left(\left[\left[{ }_{s}\left(\sigma^{*}\right)^{[0]}\right]+\left[{ }_{a}\left(\sigma^{*}\right)^{[0]}\right]\right]\left[\left[{ }_{s} \dot{J}\right]+\left[{ }_{a} \dot{j}\right]\right]\right) \\
& =\operatorname{tr}\left(\left[{ }_{s}\left(\sigma^{*}\right)^{[0]}\right]\left[{ }_{s} \dot{J}\right]\right)+\operatorname{tr}\left(\left[{ }_{a}\left(\sigma^{*}\right)^{[0]}\right]\left[{ }_{a} \dot{J}\right]\right) \\
& \operatorname{tr}\left(\left[\left(m^{*}\right)^{[0]}\right]\left[{ }^{\Theta} \dot{J}\right]\right)=\operatorname{tr}\left(\left[\left[s\left(m^{*}\right)^{[0]}\right]+\left[a\left(m^{*}\right)^{[0]}\right]\right]\left[\left[{ }^{\Theta} \dot{j}\right]+\left[{ }_{a}^{\Theta} \dot{j}\right]\right]\right) \\
& =\operatorname{tr}\left(\left[{ }_{s}\left(m^{*}\right)^{[0]}\right]\left[{ }^{\Theta} \dot{j}\right]\right)+\operatorname{tr}\left(\left[a\left(m^{*}\right)^{[0]}\right]\left[\begin{array}{l}
\Theta \\
a
\end{array}\right]\right)
\end{aligned}
$$

Using the balance of angular momenta, we can write

$$
\dot{\boldsymbol{\Theta}} \cdot\left(\boldsymbol{\nabla} \cdot\left(\boldsymbol{m}^{*}\right)^{[0]}\right)=\dot{\boldsymbol{\Theta}} \cdot\left(\boldsymbol{\epsilon}:{ }_{a}\left(\boldsymbol{\sigma}^{*}\right)^{[0]}\right)
$$

A simple calculation shows that

$$
\dot{\boldsymbol{\Theta}} \cdot\left(\boldsymbol{\epsilon}:{ }_{a}\left(\boldsymbol{\sigma}^{*}\right)^{[0]}\right)=-\operatorname{tr}\left(\left[a\left(\boldsymbol{\sigma}^{*}\right)^{[0]}\right]\left[{ }_{a} \dot{J}\right]\right)
$$

Using (3.55), the balance of angular momenta becomes

$$
\boldsymbol{\nabla} \cdot\left(\boldsymbol{m}^{*}\right)^{[0]}-\boldsymbol{\epsilon}:{ }_{a}\left(\boldsymbol{\sigma}^{*}\right)^{[0]}=0
$$

Using (3.56), (3.57), (3.58), and (3.59) in the energy equation (3.32), we obtain

$$
\begin{aligned}
& \rho_{0} \frac{D e}{D t}+\boldsymbol{\nabla} \cdot \boldsymbol{q}-\operatorname{tr}\left(\left[{ }_{s}\left(\boldsymbol{\sigma}^{*}\right)^{[0]}\right]\left[{ }_{s} \dot{J}\right]\right)-\operatorname{tr}\left(\left[{ }_{a}\left(\boldsymbol{\sigma}^{*}\right)^{[0]}\right]\left[{ }_{a} \dot{J}\right]\right) \\
& -\operatorname{tr}\left(\left[{ }_{s}\left(m^{*}\right)^{[0]}\right]\left[{ }_{s}^{\Theta} \dot{J}\right]\right)-\operatorname{tr}\left(\left[{ }_{a}\left(m^{*}\right)^{[0]}\right]\left[{ }_{a}^{\Theta} \dot{J}\right]\right)+\operatorname{tr}\left(\left[{ }_{a}\left(\sigma^{*}\right)^{[0]}\right]\left[{ }_{a} \dot{J}\right]\right)=0
\end{aligned}
$$

The fourth and last terms in (3.61) cancel and we obtain the final form of the energy equation. 


$$
\rho_{0} \frac{D e}{D t}+\boldsymbol{\nabla} \cdot \boldsymbol{q}-\operatorname{tr}\left(\left[{ }_{s}\left(\boldsymbol{\sigma}^{*}\right)^{[0]}\right][\dot{j}]\right)-\operatorname{tr}\left(\left[{ }_{s}\left(m^{*}\right)^{[0]}\right]\left[{ }_{s}^{\Theta} \dot{J}\right]\right)-\operatorname{tr}\left(\left[a\left(m^{*}\right)^{[0]}\right]\left[{ }_{a}^{\Theta} \dot{J}\right]\right)=0
$$

Also using (3.56), (3.57), (3.58), and (3.59) in the entropy inequality (3.49), we obtain

$\rho_{0}\left(\frac{D \Phi}{D t}+\eta \frac{D \theta}{D t}\right)+\frac{\boldsymbol{q} \cdot \boldsymbol{g}}{\boldsymbol{\theta}}-\operatorname{tr}\left(\left[{ }_{s}\left(\sigma^{*}\right)^{[0]}\right][s \dot{J}]\right)-\operatorname{tr}\left(\left[{ }_{s}\left(m^{*}\right)^{[0]}\right]\left[{ }_{s}^{\Theta} \dot{j}\right]\right)-\operatorname{tr}\left(\left[a\left(m^{*}\right)^{[0]}\right]\left[{ }_{a}^{\Theta} \dot{j}\right]\right) \leq 0$

The last three terms in the energy equation and the entropy inequality define rate of work conjugate pairs that are essential for deriving constitutive theories considered in followup papers for thermoelastic solids and thermoviscoelastic solids with and without memory including model problems and their solutions.

\subsection{Final mathematical model}

The final form of the conservation and balance laws: conservation of mass, balance of linear momenta, balance of angular momenta, balance of moments of moments or couples, first law of thermodynamics, and second law of thermodynamics in Lagrangian description are given in the following.

$$
\begin{gathered}
\rho_{0}=|J| \rho \\
\rho_{0} \frac{D\{v\}}{D t}-\rho_{0}\left\{F^{b}\right\}-\left[\left(\sigma^{*}\right)^{[0]}\right]^{T}\{\nabla\}=0 \\
{\left[\left(m^{*}\right)^{[0]}\right]^{T}\{\nabla\}-\boldsymbol{\epsilon}:\left[a\left(\sigma^{*}\right)^{[0]}\right]^{T}=0} \\
{\left[\left(m^{*}\right)^{[0]}\right] \neq\left[\left(m^{*}\right)^{[0]}\right]^{T}} \\
\rho_{0} \frac{D e}{D t}+\boldsymbol{\nabla} \cdot \boldsymbol{q}-\operatorname{tr}\left(\left[s\left(\sigma^{*}\right)^{[0]}\right][s \dot{s}]\right)-\operatorname{tr}\left(\left[s\left(m^{*}\right)^{[0]}\right]\left[{ }_{s}^{\Theta} \dot{j}\right]\right)-\operatorname{tr}\left(\left[a\left(m^{*}\right)^{[0]}\right]\left[{ }_{a}^{\Theta} \dot{j}\right]\right)=0
\end{gathered}
$$


$\left.\rho_{0}\left(\frac{D \Phi}{D t}+\eta \frac{D \theta}{D t}\right)+\frac{\boldsymbol{q} \cdot \boldsymbol{g}}{\boldsymbol{\theta}}-\operatorname{tr}\left(\left[s\left(\sigma^{*}\right)^{[0]}\right]\left[{ }_{s} \dot{J}\right]\right)-\operatorname{tr}\left(\left[{ }_{s}\left(m^{*}\right)^{[0]}\right]\left[{ }_{s} \dot{J}\right]\right]\right)-\operatorname{tr}\left(\left[a\left(m^{*}\right)^{[0]}\right]\left[{ }_{a} \dot{j}\right]\right) \leq 0$

In this mathematical model, the dependent variables are (numbers in the parentheses indicate the number of dependent variables)

$$
\begin{aligned}
& v_{i}(3), \quad{ }_{s}\left(\boldsymbol{\sigma}^{*}\right)^{[0]}(6), \quad{ }_{a}\left(\boldsymbol{\sigma}^{*}\right)^{[0]}(3) \\
& { }_{s}\left(\boldsymbol{m}^{*}\right)^{[0]}(6), \quad{ }_{a}\left(\boldsymbol{m}^{*}\right)^{[0]}(3), \quad e(1), \quad \boldsymbol{q}(3) \\
& \Phi(1), \quad \eta(1), \quad \theta(1): \quad \text { Total of } 28
\end{aligned}
$$

In these dependent variables, $\Phi$ and $\eta$ will be eliminated from the list of variables. Specific internal energy $e$ is a function of $\rho$ and $\theta$, i.e., $e=e(\rho, \theta)$ for the most general case of compressible solid matter, hence $e$ is also eliminated from the list of dependent variables. This leaves us with 25 dependent variables remaining in the mathematical model. We have balance of linear momenta equations (3), balance of angular momenta equations (3), energy equation (1), and from entropy inequality we have possible constitutive theories for ${ }_{s}\left(\boldsymbol{\sigma}^{*}\right)^{[0]}(6),{ }_{s}\left(\boldsymbol{m}^{*}\right)^{[0]}(6),{ }_{a}\left(\boldsymbol{m}^{*}\right)^{[0]}$ (3), and $\boldsymbol{q}$ (3), a total of 25 equations, hence the mathematical model will have closure once we have constitutive theories for ${ }_{s}\left(\boldsymbol{\sigma}^{*}\right)^{[0]},{ }_{s}\left(\boldsymbol{m}^{*}\right)^{[0]},{ }_{a}\left(\boldsymbol{m}^{*}\right)^{[0]}$, and $\boldsymbol{q}$ included with the conservation and balance laws.

\subsection{General Remarks}

In this subsection we point out and discuss some important aspects and features of the mathematical model consisting of conservation and balance laws presented in this paper.

(1) The derivation is based on the assumption of finite deformation, i.e., the deformed coordinates $\overline{\boldsymbol{x}}$ are not the same as undeformed coordinates $\boldsymbol{x}$.

(2) Since the mathematical model is in Lagrangian description and since the undeformed and the deformed configurations are not the same, an elementary tetrahedron in the reference configuration experiences finite deformation in the current configuration, hence correspondence rules are required for Cauchy stress measure in the current configuration to a stress measure in the reference configuration. In 
the work presented in this paper we have used contravariant first Piola-Kirchhoff stress tensor derived using contravariant Cauchy stress tensor in the current configuration.

(3) Stress tensor $\left(\boldsymbol{\sigma}^{*}\right)^{[0]}$ is derived based on the assumption that force $d \overline{\boldsymbol{F}}$ acting on area $d \overline{\boldsymbol{A}}$ (area of the oblique plane of the deformed tetrahedron) is same as force $d \boldsymbol{F}$ on $d \boldsymbol{A}$ (area of the oblique plane of the undeformed tetrahedron). When $d \overline{\boldsymbol{F}}=d \boldsymbol{F}$, both $d \overline{\boldsymbol{F}}$ and $d \boldsymbol{F}$ obviously have same magnitude of their components and the same directions. This assumption allows $d \boldsymbol{A}$ and $d \overline{\boldsymbol{A}}$ to be different (thus allow finite deformation), hence $d \boldsymbol{P}$ and $d \overline{\boldsymbol{P}}$ to be different as well on $d \boldsymbol{A}$ and $d \overline{\boldsymbol{A}}$.

(4) From the energy equation and entropy inequality we know that $\operatorname{tr}\left(\left[\left(\sigma^{*}\right)^{[0]}\right]^{T}[\dot{\boldsymbol{J}}]^{T}\right)$ is the rate of work. We also note that [61]

$$
\operatorname{tr}\left(\left[\left(\sigma^{*}\right)^{[0]}\right]^{T}[\dot{\boldsymbol{J}}]^{T}\right)=\operatorname{tr}\left(\left[\sigma^{[0]}\right]^{T}\left[\dot{\varepsilon}_{[0]}\right]^{T}\right)
$$

That is $\left(\boldsymbol{\sigma}^{*}\right)^{[0]}$ and $\dot{\boldsymbol{j}}$ as conjugate pair result in the same rate of work as $\boldsymbol{\sigma}^{[0]}$ and $\dot{\boldsymbol{\varepsilon}}_{[0]}$ as conjugate pair. Even though the rate of work resulting from the two conjugate pairs is same, there are some important differences. Jacobian of deformation $\boldsymbol{J}$ is a measure of deformation physics and $\overline{\boldsymbol{x}} \neq \boldsymbol{x}$ implies finite deformation, however $\boldsymbol{J}$ is not a measure of finite strain. On the other hand $\boldsymbol{\varepsilon}_{[0]}$ is a measure of finite strain. Thus, our view is that the internal polar non-classical theory in this paper is applicable for finite deformation but does not explicitly incorporate measure of finite strains in derivation of the mathematical model.

(5) The internal rotations are due to $\boldsymbol{J}$, i.e., ${ }_{a} \boldsymbol{J}$ are incorporated in the theory. This feature as presented in this paper is not dependent on the choice of conjugate pair for the rate of work.

\section{Summary and conclusions}

The development of internal polar non-classical continuum theory for isotropic, homogeneous solid continua undergoing finite deformation is presented in this paper. The Jacobian of deformation $\boldsymbol{J}$ defining stretches and internal rotations has been incorporated in its entirety in the derivation of conservation and balance laws. This aspect is absent in the corresponding finite deformation theories based on classical continuum mechanics. The theory presented in this paper considers contravariant first Piola-Kirchhoff stress tensor as a measure of stress in the derivation of the theory for 
finite deformation in which $\overline{\boldsymbol{x}} \neq \boldsymbol{x}$. The internal rotations at a material point (hence the name internal polar) are completely defined by ${ }_{a} \boldsymbol{J}$ or $\boldsymbol{\nabla} \times \boldsymbol{u}$, hence are not external degrees of freedom at a material point. This theory is obviously non-classical as it considers rotations at material points, though the rotations are internal resulting from ${ }_{a} \boldsymbol{J}$. The physics due to ${ }_{a} \boldsymbol{J}$ considered in this theory for finite deformation provides a more complete thermodynamic framework than used currently.

Due to finite deformation contravariant Cauchy moment tensor $\overline{\boldsymbol{m}}^{(0)}$ (or $\boldsymbol{m}^{(0)}$ ) has also been transformed to corresponding contravariant first Piola-Kirchhoff moment tensor $\left(\boldsymbol{m}^{*}\right)^{[0]} \cdot\left(\boldsymbol{\sigma}^{*}\right)^{[0]},\left(\boldsymbol{m}^{*}\right)^{[0]}, \boldsymbol{J}, \dot{\boldsymbol{J}},{ }^{\Theta} \boldsymbol{J}$, and ${ }^{\Theta} \boldsymbol{j}$ are various measures used in the derivation of the mathematical model. The derivation shows that:

(i) Cauchy stress tensor $\boldsymbol{\sigma}^{(0)}$ (or $\left.\overline{\boldsymbol{\sigma}}^{(0)}\right)$ is non-symmetric, hence $\left(\boldsymbol{\sigma}^{*}\right)^{[0]}$ and $\boldsymbol{\sigma}^{[0]}$ are non-symmetric as well.

(ii) Cauchy moment tensor is symmetric due to balance of moment of moments, hence $\boldsymbol{m}^{[0]}$ is symmetric but $\left(\boldsymbol{m}^{*}\right)^{[0]}$ is non-symmetric.

(iii) Conjugate pairs resulting in rate of work are decided using energy equation or the entropy inequality.

(iv) The mathematical model has closure (as many equations as the number of variables) when the constitutive theories for ${ }_{s}\left(\boldsymbol{\sigma}^{*}\right)^{[0]},{ }_{s}\left(\boldsymbol{m}^{*}\right)^{[0]},{ }_{a}\left(\boldsymbol{m}^{*}\right)^{[0]}$, and $\boldsymbol{q}$ are incorporated in the mathematical model.

The differences between gradients of internal rotations and the gradients of the infinitesimal strain tensor are clearly demonstrated to point out that the theories that utilize rotation gradients as used in this work (as warranted by the conservation and balance laws) are not strain gradient theories. In fact there does not appear to be any rational for 'strain gradients' in the conservation and balance laws. The work presented in this paper is presents a more complete thermodynamic framework in which (i) Jacobian of deformation $\boldsymbol{J}$ representing true physics of deformation is incorporated in its entirety and (ii) the deformation can be finite. The theory presented here is obviously not a stress-couple theory or a micropolar theory. Furthermore, the theory is inherently local, hence not capable of describing non-local phenomena. The thermodynamic framework presented here is valid for internal polar non-classical isotropic and homogeneous thermoelastic solids and thermoviscoelastic solids with and without memory experiencing finite deformation. Constitutive theories describing various 
types of solids and model problems showing applications of the theory derived in this paper will be presented in subsequent forthcoming papers.

\section{Acknowledgements}

The first and third authors are grateful for the support provided by their endowed professorships during the course of this research. The computational infrastructure provided by the Computational Mechanics Laboratory (CML) of the Mechanical Engineering department of the University of Kansas is gratefully acknowledged. The financial support provided to the second author by the Naval Air Warfare Center is greatly appreciated.

\section{References}

[1] K. S. Surana, M. J. Powell, and J. N. Reddy. A more complete thermodynamic framework for solid continua. Journal of Thermal Engineering, 1(1):1-13, 2015.

[2] K. S. Surana, J. N. Reddy, D. Nunez, and M. J. Powell. A polar continuum theory for solid continua. International Journal of Engineering Research and Industrial Applications, 8(2):77-106, 2015.

[3] A. C. Eringen. Mechanics of Micromorphic Materials. H. Gortler (ed.) Proc. 11th Intern. Congress. Appl. Mech., pages 131-138, 1964a.

[4] A. C. Eringen. Mechanics of Micromorphic Continua. E. Kroner(ed.) Mechanics of Generalized Continua, pages 18-35, 1968.

[5] A. C. Eringen. Theory of micropolar elasticity. H. Liebowitz (ed.) Fracture, pages 621-729, 1968.

[6] A. C. Eringen. Balance Laws of Micromorphic Mechanics. International Journal of Engineering Science, 8(10):819-828, 1970.

[7] A. C. Eringen. Theory of Thermo-Microstretch Fluids and Bubbly Liquids. International Journal of Engineering Science, 28(2):133-143, 1990.

[8] A. C. Eringen. Theory of Micropolar Elasticity. Springer, 1990.

[9] A. C. Eringen. A Unified Theory of Thermomechanical Materials. International Journal of Engineering Science, 4:179-202, 1966.

[10] A. C. Eringen. Linear Theory of Micropolar Viscoelasticity. International Journal of Engineering Science, 5:191-204, 1967.

[11] A. C. Eringen. Theory of Micromorphic Materials with Memory. International Journal of Engineering Science, 10:623-641, 1972.

[12] W. Koiter. Couple Stresses in the Theory of Elasticity, i and ii. Nederl. Akad. Wetensch. Proc. Ser. B, 67:17-44, 1964. 
[13] W. Oevel and J. Schröter. Balance Equations for Micromorphic Materials. Journal of Statistical Physics, 25(4):645-662, 1981.

[14] J. N. Reddy. Nonlocal Theories for Bending, Buckling and Vibration of Beams. International Journal of Engineering Science, 45:288-307, 2007.

[15] J. N. Reddy and S. D. Pang. Nonlocal Continuum Theories of Beams for the Analysis of Carbon Nanotubes. Journal of Applied Physics, 103, 023511, 2008.

[16] J. N. Reddy. Nonlocal nonlinear Formulations for Bending of Classical and Shear Deformation Theories of Beams and Plates. International Journal of Engineering Science, 48:1507-1518, 2010.

[17] P. Lu, P. Q. Zhang, H. P. Leo, C. M. Wang, and J. N. Reddy. Nonlocal Elastic Plate Theories. Proceedings of Royal Society A, 463:3225-3240, 2007.

[18] J. F. C. Yang and R. S. Lakes. Experimental Study of Micropolar and Couple Stress Elasticity in Compact Bone in Bending. Journal of Biomechanics, 15(2):91-98, 1982.

[19] V. A. Lubarda and X. Markenscoff. Conservation Integrals in Couple Stress Elasticity. Journal of Mechanics and Physics of Solids, 48:553-564, 2000.

[20] H. M. Ma, X. L. Gao, and J. N. Reddy. A Microstructure-dependent Timoshenko Beam Model Based on a Modified Couple Stress Theory. Journal of Mechanics and Physics of Solids, 56:3379-3391, 2008.

[21] H. M. Ma, X. L. Gao, and J. N. Reddy. A Nonclassical Reddy-Levinson Beam Model Based on Modified Couple Stress Theory. Journal of Multiscale Computational Engineering, 8(2):167-180, 2010.

[22] J. N. Reddy. Microstructure Dependent Couple Stress Theories of Functionally Graded Beams. Journal of Mechanics and Physics of Solids, 59:2382-2399, 2011.

[23] J. N. Reddy and A. Arbind. Bending Relationship Between the Modified Couple Stress-based Functionally Graded Timoshenko Beams and Homogeneous Bernoulli-Euler Beams. Ann. Solid Struc. Mech., 3:15-26, 2012.

[24] A. R. Srinivasa and J. N. Reddy. A Model for a Constrained, Finitely Deforming Elastic Solid with Rotation Gradient Dependent Strain Energy and its Specialization to Von Kármán Plates and Beams. Journal of Mechanics and Physics of Solids, 61(3):873-885, 2013.

[25] R. J. Mora and A. M. Waas. Evaluation of the Micropolar Elasticity Constants for Honeycombs. Acta Mechanica, 192:1-16, 2007.

[26] P. R. Onck. Cosserat Modeling of Cellular Solids. C. R. Mecanique, 330:717-722, 2002.

[27] P. H. Segerstad, S. Toll, and R. Larsson. A Micropolar Theory for the Finite Elasticity of Open-cell Cellular Solids. Proceedings of Royal Society A, 465:843-865, 2009. 
[28] H. Altenbach and V. A. Eremeyev. On the linear theory of micropolar plates. ZAMM-Journal of Applied Mathematics and Mechanics/Zeitschrift für Angewandte Mathematik und Mechanik, 89(4):242256, 2009.

[29] H. Altenbach and V. A. Eremeyev. Strain rate tensors and constitutive equations of inelastic micropolar materials. International Journal of Plasticity, 63:3-17, 2014.

[30] H. Altenbach, V. A. Eremeyev, L. P. Lebedev, and L. A. Rendón. Acceleration waves and ellipticity in thermoelastic micropolar media. Archive of Applied Mechanics, 80(3):217-227, 2010.

[31] H. Altenbach, G. A. Maugin, and V. Erofeev. Mechanics of generalized continua, volume 7. Springer, 2011.

[32] H. Altenbach, K. Naumenko, and P. A. Zhilin. A micro-polar theory for binary media with application to phase-transitional flow of fiber suspensions. Continuum Mechanics and Thermodynamics, 15(6):539-570, 2003.

[33] F. Ebert. A similarity solution for the boundary layer flow of a polar fluid. The Chemical Engineering Journal, 5(1):85-92, 1973.

[34] V. A. Eremeyev, L. P. Lebedev, and H. Altenbach. Kinematics of micropolar continuum. In Foundations of Micropolar Mechanics, pages 11-13. Springer, 2013.

[35] V. A. Eremeyev and W. Pietraszkiewicz. Material symmetry group and constitutive equations of anisotropic Cosserat continuum. Generalized Continua As Models for Materials, page 10, 2012.

[36] V. A. Eremeyev and W. Pietraszkiewicz. Material symmetry group of the non-linear polar-elastic continuum. International Journal of Solids and Structures, 49(14):1993-2005, 2012.

[37] E. F. Grekova. Ferromagnets and Kelvin's medium: Basic equations and wave processes. Journal of Computational Acoustics, 9(02):427-446, 2001.

[38] E. F. Grekova. Linear reduced Cosserat medium with spherical tensor of inertia, where rotations are not observed in experiment. Mechanics of Solids, 47(5):538-543, 2012.

[39] E. F. Grekova, M. A. Kulesh, and G. C. Herman. Waves in linear elastic media with microrotations, part 2: Isotropic reduced Cosserat model. Bulletin of the Seismological Society of America, 99(2B):1423$1428,2009$.

[40] G. Grioli. Linear micropolar media with constrained rotations. In Micropolar Elasticity, pages 45-71. Springer, 1974.

[41] E. F. Grekova and G. A. Maugin. Modelling of complex elastic crystals by means of multi-spin micromorphic media. International Journal of Engineering Science, 43(5):494-519, 2005.

[42] G. Grioli. Microstructures as a refinement of cauchy theory. problems of physical concreteness. Continuum Mechanics and Thermodynamics, 15(5):441-450, 2003.

[43] J. Altenbach, H. Altenbach, and V. A. Eremeyev. On generalized Cosserat-type theories of plates and shells: a short review and bibliography. Archive of Applied Mechanics, 80(1):73-92, 2010. 
[44] M. Lazar and G. A. Maugin. Nonsingular stress and strain fields of dislocations and disclinations in first strain gradient elasticity. International Journal of Engineering Science, 43(13):1157-1184, 2005.

[45] M. Lazar and G.A. Maugin. Defects in gradient micropolar elasticity âĂŤI: screw dislocation. Journal of the Mechanics and Physics of Solids, 52(10):2263-2284, 2004.

[46] G. A. Maugin. A phenomenological theory of ferroliquids. International Journal of Engineering Science, 16(12):1029-1044, 1978.

[47] G. A. Maugin. Wave motion in magnetizable deformable solids. International Journal of Engineering Science, 19(3):321-388, 1981.

[48] G. A. Maugin. On the structure of the theory of polar elasticity. Philosophical Transactions of the Royal Society of London. Series A: Mathematical, Physical and Engineering Sciences, 356(1741):1367-1395, 1998.

[49] W. Pietraszkiewicz and V. A. Eremeyev. On natural strain measures of the non-linear micropolar continuum. International Journal of Solids and Structures, 46(3):774-787, 2009.

[50] E. Cosserat and F. Cosserat. Théorie des corps déformables. Paris, 1909.

[51] A. V. Zakharov and E. L. Aero. Statistical mechanical theory of polar fluids for all densities. Physica A: Statistical Mechanics and its Applications, 160(2):157-165, 1989.

[52] A. E. Green. Micro-materials and multipolar continuum mechanics. International Journal of Engineering Science, 3(5):533-537, 1965.

[53] A. E. Green and R. S. Rivlin. The relation between director and multipolar theories in continuum mechanics. Zeitschrift für angewandte Mathematik und Physik ZAMP, 18(2):208-218, 1967.

[54] A. E. Green and R. S. Rivlin. Multipolar continuum mechanics. Archive for Rational Mechanics and Analysis, 17(2):113-147, 1964.

[55] L. C. Martins, R. F. Oliveira, and P. Podio-Guidugli. On the vanishing of the additive measures of strain and rotation for finite deformations. Journal of elasticity, 17(2):189-193, 1987.

[56] L. C. Martins and P. Podio-Guidugli. On the local measures of mean rotation in continuum mechanics. Journal of elasticity, 27(3):267-279, 1992.

[57] W. Nowacki. Theory of micropolar elasticity. Springer, 1970.

[58] F. A. C. M. Yang, A. C. M. Chong, D. C. C. Lam, and P. Tong. Couple stress based strain gradient theory for elasticity. International Journal of Solids and Structures, 39(10):2731-2743, 2002.

[59] A. C. Eringen. Mechanics of continua. Huntington, NY, Robert E. Krieger Publishing Co., 1980. 606 p., $1,1980$.

[60] J. N. Reddy. An introduction to continuum mechanics. Cambridge University Press, Cambridge, 2013.

[61] K. S. Surana. Advanced Mechanics of Continua. CRC/Taylor and Francis, Boca Raton, FL, 2015. 
[62] K. S. Surana, Y. Ma, A. Romkes, and J. N. Reddy. The rate constitutive equations and their validity for progressively increasing deformation. Mechanics of Advanced Materials and Structures, 17(7):509$533,2010$.

[63] P. Steinmann. A micropolar theory of finite deformation and finite rotation multiplicative elastoplasticity. International Journal of Solids and Structures, 31(8):1063-1084, 1994.

[64] A. R. Srinivasa and J. N. Reddy. A model for a constrained, finitely deforming, elastic solid with rotation gradient dependent strain energy, and its specialization to von Kármán plates and beams. Journal of Mechanics and Physics of Solids, 61(3):873-885, 2013.

[65] P. H. Segerstad, S. Toll, and R. Larsson. A micropolar theory for the finite elasticity of open-cell cellular solids. Proceedings of the Royal Society A, 465:843-865, 2008.

[66] R. T. Shield. The rotation associated with large strains. SIAM Journal on Applied MAthematics, 25(3):483-491, 1973. 